\title{
Empirical Best Prediction Under Unit-Level Logit Mixed Models
}

\author{
Tomáš Hobza ${ }^{1}$ and Domingo Morales ${ }^{2}$
}

\begin{abstract}
The article applies unit-level logit mixed models to estimating small-area weighted sums of probabilities. The model parameters are estimated by the method of simulated moments (MSM). The empirical best predictor (EBP) of weighted sums of probabilities is calculated and compared with plug-in estimators. An approximation to the mean-squared error (MSE) of the EBP is derived and a bias-corrected MSE estimator is given and compared with parametric bootstrap alternatives. Some simulation experiments are carried out to study the empirical behavior of the model parameter MSM estimators, the EBP and plug-in estimators and the MSE estimators. An application to the estimation of poverty proportions in the counties of the region of Valencia, Spain, is given.
\end{abstract}

Key words: Poverty; method of moments; logit mixed models; empirical best predictor; mean-squared error; bootstrap.

\section{Introduction}

This article deals with the estimation of weighted sums of probabilities in domains where the sample size is not large enough to obtain reliable direct estimates. Small-area estimation (SAE) deals with this problem by introducing model-based or model-assisted estimators. See the monographs of Rao (2003) and Rao and Molina (2015), and the reviews of Ghosh and Rao (1994), Rao (1999), Pfeffermann (2002, 2013), and Jiang and Lahiri (2006) for an introduction to SAE.

The binomial-logit mixed models are generalized linear mixed models (GLMM) that take into account the between-domains variability that it is not explained through auxiliary variables by introducing random effects. The random effects are usually assumed to be normally distributed. Inferences based on GLMMs have some computational difficulties because the likelihood may involve high-dimensional integrals which cannot be evaluated analytically. This article uses the method of simulated moments (MSM), introduced by Jiang (1998), to fit the proposed model. This method approximates the method of moments (MM), is computationally attractive, and gives consistent estimators of model parameters.

1 Department of Mathematics, Czech Technical University in Prague, Trojanova 13, 12000 Prague 2, Czech Republic. Email: hobza@fjfi.cvut.cz

2 Operations Research Center, Miguel Hernández University of Elche, Avda. de la Universidad s/n, 03202 Elche, Spain. Email: d.morales@umh.es

Acknowledgments: The author thanks the Office of Social, Demographic and Economic Statistics of the Valencian Government for providing the real data employed in the application of this article. This study was partially supported by the Spanish grant MTM2015-64842-P and by the Czech grant SGS15/214/OHK4/3T/14. 
The article derives empirical best predictors (EBP) for estimating weighted sums of probabilities, where the weights are known quantities taken from administrative registers or census files. This is to say, the term "weighting" is distinct from sample survey weighting based on the survey design. The EBPs are sums of predicted values of probabilities (sometimes called soft estimates) rather than sums of target-variable predictions that must be 0 or 1 (hard estimates). The statistical methodology is taken and adapted from Jiang and Lahiri (2001) and Jiang (2003), where EBPs of functions of fixed effects and small-area-specific random effects were developed in the context of logistic mixed models and GLMM respectively. Furthermore, plug-in estimators are considered and empirically studied in simulation experiments.

The MSE is a standard accuracy measure for point estimators. Jiang and Lahiri (2001) and Jiang (2003) studied the approximation of the MSE of the EBP in the context of binary data and GLMM. Their approach is based on Taylor series expansions. They further gave a second-order bias-corrected estimator of the MSE. We adapt the MSE calculations given by these authors to the case of EBPs of weighted sums of probabilities. We give two analytical estimators of the MSE approximation, without and with a bias-correction term. As these MSE estimators are computationally expensive in practice, we consider the parametric bootstrap estimator introduced by Gonzalez-Manteiga et al. (2007) in the context of logistic mixed models. This approach was later extended by GonzalezManteiga et al. (2008a,b) to nested error regression models and to multivariate area-level models respectively. As the simple parametric bootstrap method tends to underestimate the MSE when domain sizes are too small, we also give a double-bootstrap bias-corrected estimator by following Hall and Maiti (2006a,b).

Jiang and Lahiri (2001) and Jiang (2003) introduced the basic statistical methodology for EBPs of functions of fixed and random effects. They also studied the large sample properties of the EBPs and MSE estimators. However, they did not carry out simulation experiments to empirically investigate the behavior of the EBPs and MSE estimators in the standard small-area estimation setup, that is when the domain sample sizes are small. They also did not present applications to real data. This article in part covers this gap.

We carry out simulation experiments to investigate the behavior of the EBPs of weighted sums of probabilities and the corresponding MSE estimators. We investigate computational and numerical issues appearing in the implementation of the EBP methodology. The article also presents an application to 2012 data from the Spanish living conditions survey (SLCS2012) in the region of Valencia. The target of the application is the estimation of poverty proportions at county level.

Other SAE models for the estimation of poverty proportions are currently available. Without being exhaustive, we cite some basic references here. Chambers et al. (2012) introduce an M-quantile regression approach for binary data. Farrell et al. (1997) give bootstrap adjustments for empirical Bayes interval estimates of small-area proportions. Malec et al. (1997) gave some small-area inference methods for binary variables. By using unit-level linear mixed models, Molina et al. (2014) and Molina and Rao (2010) give several procedures for estimating poverty indicators. There is also Elbers et al. (2003) and its extensions, who use full unit record census data, rather than building a model-based census substitute from cross tabulations. Based on temporal and spatio-temporal area-level 
models, Esteban et al. (2012a,b), Marhuenda et al. (2013), and Morales et al. (2015) give EBLUPs of poverty proportions.

The article is organized as follows. Section 2 introduces the unit-level binomial-logit mixed model and the employed fitting algorithm. Section 3 presents the EBPs (which in this article are synthetic because they assume random effects given small area are zero mean) and the plug-in estimators of weighted sums of probabilities. Section 4 gives an approximation to the MSE of the EBP and four estimators. The first two MSE estimators are plug-in derivations of the MSE approximation, without and with bias correction term. The third and fourth MSE estimators are based on parametric bootstrap. Section 5 presents three simulation experiments. The first simulation studies the behavior of the MSM fitting algorithm. The second simulation compares the performances of the EBPs and the plug-in estimators. The third simulation deals with the MSE estimators proposed in Section 4. All use relatively small populations in comparison with real-life applications. Section 6 applies the developed methodology to data from the SLCS2012 using model-based synthetic estimators with small-area level random effects only. The target is the estimation of poverty proportions at county level. Section 7 gives a discussion and some conclusions.

The article contains four appendixes. Appendix A.1 gives the components of the updating equation of the MSM algorithm. Appendix A.2 contains the proof of Proposition 4.1. Appendix A.3 presents some routines for MSE calculation and Appendix A.4 describes approximations of some derivatives needed for MSE calculation.

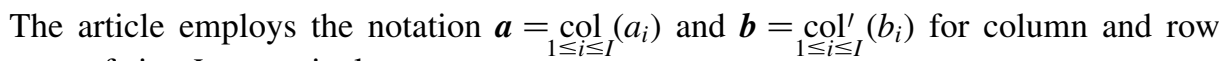
vectors of size $I$ respectively.

\section{The Model}

This section introduces a unit-level binomial-logit mixed model and its MSM fitting algorithm. Let $D$ be the number of small areas or domains, with $d=1, \ldots, D$. Let $\left\{v_{d}: d=1, \ldots, D\right\}$ be a set of independent and identically distributed (i.i.d.) $N(0,1)$ random effects, which is a reasonable assumption for sufficiently large domains. Note that in this model, random effects at finer levels are not considered. In matrix notation, we have $\boldsymbol{v}=\left(v_{1}, \ldots, v_{D}\right)^{\prime} \sim N_{D}\left(\mathbf{0}, \boldsymbol{I}_{D}\right)$, where $\boldsymbol{I}_{D}$ is the $D \times D$ unit matrix. The target variable $y_{d j}$ represents the $j$ th sample observation from domain $d$ and its distribution, conditioned to the random effect $v_{d}$, is

$$
\left.y_{d j}\right|_{v_{d}} \sim \operatorname{Bin}\left(m_{d j}, p_{d j}\right), \quad d=1, \ldots, D, \quad j=1, \ldots, n_{d},
$$

where $m_{d j}$ is a known size parameter. The binomial distribution is typically employed for counting numbers of successes. In official statistics, the sample units can be individuals, households, companies and so on. For individuals, we can investigate the presence of a characteristic of interest and the corresponding size parameter is one. For households and companies, we might be interested in counting the number of household members with lactose intolerance or the number of employees with a high salary. Then the size parameter is the number of household members or company workers, respectively. In all cases, we can assume that the size parameters are known quantities for the sampled elements. Note that in the model there is assumed to be no clustering at survey primary sampling unit level or at household level. 
For the natural parameter, we assume

$$
\eta_{d j}=\log \frac{p_{d j}}{1-p_{d j}}=\boldsymbol{x}_{d j} \boldsymbol{\beta}+\phi v_{d}, \quad d=1, \ldots, D, \quad j=1, \ldots, n_{d},
$$

where $\phi>0$ is a variance parameter, $\boldsymbol{\beta}=\underset{1 \leq k \leq p}{\operatorname{col}}\left(\beta_{k}\right)$ is the column vector of regression parameters and $\boldsymbol{x}_{d j}=\underset{1 \leq k \leq p}{\operatorname{col} l^{\prime}}\left(x_{d j k}\right)$ is a row vector of auxiliary variables. We also assume that the $y_{d j}$ 's are independent conditioned to $v$. It holds that

$$
\begin{aligned}
P\left(y_{d j} \mid \boldsymbol{v}\right) & =P\left(y_{d j} \mid v_{d}\right)=\left(\begin{array}{c}
m_{d j} \\
y_{d j}
\end{array}\right) p_{d j}^{y_{d j}}\left(1-p_{d j}\right)^{m_{d j}-y_{d j}}, \\
p_{d j} & =\frac{\exp \left\{\boldsymbol{x}_{d j} \beta+\phi v_{d}\right\}}{1+\exp \left\{\boldsymbol{x}_{d j} \beta+\phi v_{d}\right\}} .
\end{aligned}
$$

Let us define $\boldsymbol{y}=\underset{1 \leq d \leq D}{\operatorname{col}}\left(\boldsymbol{y}_{d}\right)$, where $\boldsymbol{y}_{d}=\underset{1 \leq j \leq n_{d}}{\operatorname{col}}\left(y_{d j}\right)$. The marginal distribution of $\boldsymbol{y}$ is

$$
P(\boldsymbol{y})=\prod_{d=1}^{D} \int_{R} P\left(\boldsymbol{y}_{d} \mid v_{d}\right) f\left(v_{d}\right) d v_{d}
$$

where $f$ is the standard normal probability density function and

$$
\begin{aligned}
& P\left(\boldsymbol{y}_{d} \mid v_{d}\right)=\prod_{j=1}^{n_{d}} P\left(y_{d j} \mid v_{d}\right)=\prod_{j=1}^{n_{d}}\left(\begin{array}{c}
m_{d j} \\
y_{d j}
\end{array}\right) \frac{\exp \left\{y_{d j}\left(\boldsymbol{x}_{d j} \boldsymbol{\beta}+\phi v_{d}\right)\right\}}{\left[1+\exp \left\{\boldsymbol{x}_{d j} \boldsymbol{\beta}+\phi v_{d}\right\}\right]^{m_{d j}}} \\
& =\exp \left\{\sum_{j=1}^{n_{d}} \log \left(\begin{array}{c}
m_{d j} \\
y_{d j}
\end{array}\right)+\sum_{j=1}^{n_{d}} y_{d j}\left(\boldsymbol{x}_{d j} \boldsymbol{\beta}+\phi v_{d}\right)-\sum_{j=1}^{n_{d}} m_{d j} \log \left[1+\exp \left\{\boldsymbol{x}_{d j} \boldsymbol{\beta}+\phi v_{d}\right\}\right]\right\} .
\end{aligned}
$$

Let $\boldsymbol{\theta}=\left(\boldsymbol{\beta}^{\prime}, \phi\right)^{\prime}$ be the vector of model parameters. To fit the unit-level binomial-logit mixed model, we employ the MSM algorithm suggested by Jiang (1998). A natural set of equations for applying the method of moments is

$$
\begin{aligned}
& 0=f_{k}(\boldsymbol{\theta})=M_{k}(\boldsymbol{\theta})-\hat{M}_{k}=\sum_{d=1}^{D} \sum_{j=1}^{n_{d}} E_{\boldsymbol{\theta}}\left[y_{d j}\right] x_{d j k}-\sum_{d=1}^{D} \sum_{j=1}^{n_{d}} y_{d j} x_{d j k}, \quad k=1, \ldots p, \\
& 0=f_{p+1}(\boldsymbol{\theta})=M_{p+1}(\boldsymbol{\theta})-\hat{M}_{p+1}=\sum_{d=1}^{D} E_{\boldsymbol{\theta}}\left[y_{d}^{2}\right]-\sum_{d=1}^{D} y_{d .}^{2},
\end{aligned}
$$

where $y_{d}=\sum_{j=1}^{n_{d}} y_{d j}$. For solving this system of nonlinear equations, which involve matching mean and sums of squares, the Newton-Raphson updating formula is

$$
\boldsymbol{\theta}^{(r+1)}=\boldsymbol{\theta}^{(r)}-\boldsymbol{H}^{-1}\left(\boldsymbol{\theta}^{(r)}\right) \boldsymbol{f}\left(\boldsymbol{\theta}^{(r)}\right),
$$

where $\theta_{1}=\beta_{1}, \ldots, \theta_{p}=\beta_{p}, \theta_{p+1}=\phi$ and

$$
\boldsymbol{\theta}=\operatorname{col}_{1 \leq k \leq p+1}\left(\theta_{k}\right), \quad \boldsymbol{f}(\boldsymbol{\theta})=\operatorname{col}_{1 \leq k \leq p+1}\left(f_{k}(\boldsymbol{\theta})\right), \quad \boldsymbol{H}(\boldsymbol{\theta})=\left(\frac{\partial f_{k}(\boldsymbol{\theta})}{\partial \theta_{\ell}}\right)_{k, \ell=1, \ldots, p+1} .
$$

Appendix A.1 gives the components of vector $\boldsymbol{f}$ and matrix $\boldsymbol{H}$ appearing in (2.4). As the expectations appearing in the components cannot be explicitly calculated, they are 
approximated by Monte Carlo simulation. As the algorithm seed for $\boldsymbol{\beta}$, we use $\boldsymbol{\beta}^{(0)}=\tilde{\boldsymbol{\beta}}$, where $\tilde{\boldsymbol{\beta}}$ is the maximum-likelihood estimator under the model without random effects. In that model, the natural parameters are

$$
\eta_{d j}=x_{d j} \beta, \quad d=1, \ldots, D, \quad j=1, \ldots, n_{d} .
$$

Concerning the variance parameter, we use

$$
\begin{gathered}
\phi^{(0)}=\left(\frac{1}{n} \sum_{d=1}^{D} \sum_{j=1}^{n_{d}}\left(\tilde{\eta}_{d j}-\hat{\eta}_{d .}^{d i r}\right)^{2}\right)^{1 / 2}, \quad \hat{\eta}_{d .}^{d i r}=\log \frac{\hat{p}_{d .}^{d i r}}{1-\hat{p}_{d .}^{d i r}} \\
\hat{p}_{d .}^{d i r}=\frac{1}{n_{d}} \sum_{j=1}^{n_{d}} \frac{y_{d j}}{m_{d j}}, \quad \tilde{\eta}_{d j}=\boldsymbol{x}_{d j} \tilde{\boldsymbol{\beta}}
\end{gathered}
$$

where $n=\sum_{d=1}^{D} n_{d}$ is the total sample size. A bootstrap algorithm to estimate $\operatorname{var}(\hat{\boldsymbol{\theta}})$ is

1. Fit the Model (2.1)-(2.2) to the sample and calculate $\hat{\boldsymbol{\theta}}$.

2. Generate bootstrap samples $\left\{y_{d j}^{(b)}: d=1, \ldots, D, j=1, \ldots, n_{d}\right\}, b=1, \ldots, B$, from the fitted model. Fit the Model $(2.1)-(2.2)$ to the bootstrap samples and calculate $\hat{\boldsymbol{\theta}}^{(b)}, b=1, \ldots, B$, and $\overline{\boldsymbol{\theta}}=\frac{1}{B} \sum_{b=1}^{B} \hat{\boldsymbol{\theta}}^{(b)}$.

3. Output: $\widehat{\operatorname{var}}_{B}(\hat{\boldsymbol{\theta}})=\frac{1}{B} \sum_{b=1}^{B}\left(\hat{\boldsymbol{\theta}}^{(b)}-\overline{\boldsymbol{\theta}}\right)\left(\hat{\boldsymbol{\theta}}^{(b)}-\overline{\boldsymbol{\theta}}\right)^{\prime}$.

\section{Empirical Best Prediction}

Let us assume that the unit-level binomial-logit mixed model (2.1)-(2.2), with random effects at domain level only, holds for all the units of a population $U$ partitioned into $D$ domains $U_{1}, \ldots, U_{D}$ of sizes $N_{1}, \ldots, N_{D}$. The best predictor $(\mathrm{BP})$ of $p_{d j}=p_{d j}\left(\boldsymbol{\theta}, v_{d}\right)$ and of the sum of probabilities $\mu_{d}=\mu_{d}\left(\boldsymbol{\theta}, v_{d}\right)=\sum_{j=1}^{N_{d}} p_{d j}$ is

$$
\begin{aligned}
& \hat{p}_{d j}(\boldsymbol{\theta})=E_{\boldsymbol{\theta}}\left[p_{d j} \mid \boldsymbol{y}_{d}\right]=\frac{\int_{R} \frac{\exp \left\{\boldsymbol{x}_{d j} \boldsymbol{\beta}+\phi v_{d}\right\}}{1+\exp \left\{\boldsymbol{x}_{d j} \boldsymbol{\beta}+\phi v_{d}\right\}} P\left(\boldsymbol{y}_{d} \mid v_{d}\right) f\left(v_{d}\right) d v_{d}}{\int_{R} P\left(\boldsymbol{y}_{d} \mid v_{d}\right) f\left(v_{d}\right) d v_{d}}=\frac{A_{d j}}{C_{d}}, \\
& \hat{\mu}_{d}(\boldsymbol{\theta})=\sum_{j=1}^{N_{d}} \hat{p}_{d j}(\boldsymbol{\theta}),
\end{aligned}
$$

where $f$ is the standard normal probability density function and

$$
\begin{gathered}
A_{d j}=\int_{R} \frac{\exp \left\{\boldsymbol{x}_{d j} \boldsymbol{\beta}+\phi v_{d}\right\}}{1+\exp \left\{\boldsymbol{x}_{d j} \boldsymbol{\beta}+\phi v_{d}\right\}} \exp \left\{\phi y_{d .} v_{d}-\sum_{i=1}^{n_{d}} m_{d i} \log \left[1+\exp \left\{\boldsymbol{x}_{d i} \boldsymbol{\beta}+\phi v_{d}\right\}\right]\right\} f\left(v_{d}\right) d v_{d}, \\
C_{d}=\int_{R} \exp \left\{\phi y_{d .} v_{d}-\sum_{i=1}^{n_{d}} m_{d i} \log \left[1+\exp \left\{\boldsymbol{x}_{d i} \boldsymbol{\beta}+\phi v_{d}\right\}\right]\right\} f\left(v_{d}\right) d v_{d} .
\end{gathered}
$$

The EBP of $p_{d j}$ and $\mu_{d}$ are $\hat{p}_{d j}(\hat{\boldsymbol{\theta}})$ and $\hat{\mu}_{d}(\hat{\boldsymbol{\theta}})$ and they can be approximated by Monte Carlo simulation. If at least one of the auxiliary variables is continuous, the calculation of $\hat{\mu}_{d}(\hat{\boldsymbol{\theta}})$ requires the availability of census files with the values of $\boldsymbol{x}_{d j}$ for all the units $j \in U_{d}$. Two data files (survey and census), with the same auxiliary variables, are then needed to 
calculate the EBP of $\mu_{d}$ in this setup. In the real data case of Section 6, the $x$-values are only available for the sample units. The nonavailability of census data is the standard situation for the living conditions public statistics in most countries. This is why we study the special case where the covariates are categorical and take a finite number of values. For this last case, the EBPs can be calculated without having a full unit-record census file, with the caveats that using various cross-tabulations implies a specific, untested census structure, and excludes the use of continuous variables in the survey-based binomial-logit mixed model. However, the external-to-sample information can be obtained more easily from cross tabulations, as detailed unit-record data have limited availability.

Let us assume that $\boldsymbol{x}_{d j} \in\left\{z_{1}, \ldots, z_{K}\right\}$ for all $d$ and $j$. Define

$$
\bar{\mu}_{d}=\frac{\mu_{d}}{N_{d}}, \quad \mu_{d}=\sum_{j=1}^{N_{d}} p_{d j}=\sum_{k=1}^{K} N_{d k} q_{d k}, \quad q_{d k}=\frac{\exp \left\{\boldsymbol{z}_{k} \boldsymbol{\beta}+\phi v_{d}\right\}}{1+\exp \left\{\boldsymbol{z}_{k} \boldsymbol{\beta}+\phi v_{d}\right\}}
$$

and $N_{d k}=\#\left\{j \in U_{d}: \boldsymbol{x}_{d j}=\boldsymbol{z}_{k}\right\}$ is the known size of the covariate class $\boldsymbol{z}_{k}$ at the domain $d$. The target of this section is the estimation of the parameters defined in (3.2).

The BP of the random effect, $v_{d}$, and of the weighted sums of probabilities, $\mu_{d}$ and $\bar{\mu}_{d}$, are

$$
\begin{gathered}
\hat{v}_{d}(\boldsymbol{\theta})=E_{\boldsymbol{\theta}}\left[v_{d} \mid \boldsymbol{y}_{d}\right]=\frac{\int_{R} v_{d} P\left(\boldsymbol{y}_{d} \mid v_{d}\right) f\left(v_{d}\right) d v_{d}}{\int_{R} P\left(\boldsymbol{y}_{d} \mid v_{d}\right) f\left(v_{d}\right) d v_{d}}=\frac{A_{d}^{v}}{C_{d}}, \\
\hat{\mu}_{d}(\boldsymbol{\theta})=E_{\boldsymbol{\theta}}\left[\mu_{d} \mid \boldsymbol{y}_{d}\right]=\sum_{k=1}^{K} N_{d k} E_{\boldsymbol{\theta}}\left[q_{d k} \mid y_{d}\right]=: \psi_{d}\left(y_{d}, \boldsymbol{\theta}\right), \quad \hat{\bar{\mu}}_{d}(\boldsymbol{\theta})=\frac{\hat{\mu}_{d}(\boldsymbol{\theta})}{N_{d}},
\end{gathered}
$$

where the symbol $=$ : stands for notation,

$$
E_{\boldsymbol{\theta}}\left[q_{d k} \mid \boldsymbol{y}_{d}\right]=\frac{\int_{R} \frac{\exp \left\{\boldsymbol{z}_{k} \boldsymbol{\beta}+\boldsymbol{\phi} v_{d}\right\}}{1+\exp \left\{\boldsymbol{z}_{k} \boldsymbol{\beta}+\phi v_{d}\right\}} P\left(\boldsymbol{y}_{d} \mid v_{d}\right) f\left(v_{d}\right) d v_{d}}{\int_{R} P\left(\boldsymbol{y}_{d} \mid v_{d}\right) f\left(v_{d}\right) d v_{d}}=\frac{A_{d k}^{z}}{C_{d}}
$$

and

$$
\begin{aligned}
A_{d k}^{z} & =\int_{R} \frac{\exp \left\{z_{k} \boldsymbol{\beta}+\phi v_{d}\right\}}{1+\exp \left\{z_{k} \boldsymbol{\beta}+\phi v_{d}\right\}} \exp \left\{\phi y_{d .} v_{d}-\sum_{i=1}^{n_{d}} m_{d i} \log \left[1+\exp \left\{\boldsymbol{x}_{d i} \boldsymbol{\beta}+\phi v_{d}\right\}\right]\right\} f\left(v_{d}\right) d v_{d}, \\
A_{d}^{v} & =\int_{R} v_{d} \exp \left\{\phi y_{d .} v_{d}-\sum_{i=1}^{n_{d}} m_{d i} \log \left[1+\exp \left\{\boldsymbol{x}_{d i} \boldsymbol{\beta}+\phi v_{d}\right\}\right]\right\} f\left(v_{d}\right) d v_{d} \\
C_{d} & =\int_{R} \exp \left\{\phi y_{d .} v_{d}-\sum_{i=1}^{n_{d}} \nu_{d i} \log \left[1+\exp \left\{\boldsymbol{x}_{d i} \boldsymbol{\beta}+\phi v_{d}\right\}\right]\right\} f\left(v_{d}\right) d v_{d} .
\end{aligned}
$$

The EBPs of $v_{d}, \mu_{d}$ and $\bar{\mu}_{d}$ are $\hat{v}_{d}=\hat{v}_{d}(\hat{\boldsymbol{\theta}}), \hat{\mu}_{d}(\hat{\boldsymbol{\theta}})=\psi_{d}\left(y_{d}, \hat{\boldsymbol{\theta}}\right)$ and $\hat{\bar{\mu}}_{d}=\hat{\mu}_{d}(\hat{\boldsymbol{\theta}}) / N_{d}$, respectively. They can be approximated by Monte Carlo simulation. For $\hat{v}_{d}(\hat{\boldsymbol{\theta}})$ and $\hat{\mu}_{d}(\hat{\boldsymbol{\theta}})$, the algorithm steps are 
1. Estimate $\hat{\boldsymbol{\theta}}=\left(\hat{\boldsymbol{\beta}}^{\prime}, \hat{\boldsymbol{\phi}}\right)^{\prime}$ and generate $v_{d}^{(s)}$ i.i.d. $N(0,1), v_{d}^{(S+s)}=-v_{d}^{(s)}, s=1, \ldots, S$, $d=1, \ldots, D$.

2. Calculate $\hat{v}_{d}=\hat{A}_{d}^{v} / \hat{C}_{d}$ and $\hat{\mu}_{d}(\hat{\boldsymbol{\theta}})=\sum_{k=1}^{K} N_{d k} \hat{q}_{d k}$, where $\hat{q}_{d k}=\hat{A}_{d k}^{z} / \hat{C}_{d}$ and

$$
\begin{aligned}
& \hat{A}_{d k}^{z}=\frac{1}{2 S} \sum_{s=1}^{2 S} \frac{\exp \left\{\boldsymbol{z}_{k} \hat{\boldsymbol{\beta}}+\hat{\boldsymbol{\phi}} v_{d}^{(s)}\right\}}{1+\exp \left\{\boldsymbol{z}_{k} \hat{\boldsymbol{\beta}}+\hat{\boldsymbol{\phi}} v_{d}^{(s)}\right\}} \exp \left\{\hat{\boldsymbol{\phi}} y_{d .} v_{d}^{(s)}-\sum_{i=1}^{n_{d}} m_{d i} \log \left[1+\exp \left\{\boldsymbol{x}_{d i} \hat{\boldsymbol{\beta}}+\hat{\boldsymbol{\phi}} v_{d}^{(s)}\right\}\right]\right\}, \\
& \hat{A}_{d}^{v}=\frac{1}{2 S} \sum_{s=1}^{2 S} v_{d}^{(s)} \exp \left\{\hat{\boldsymbol{\phi}} y_{d .} v_{d}^{(s)}-\sum_{i=1}^{n_{d}} m_{d i} \log \left[1+\exp \left\{\boldsymbol{x}_{d i} \hat{\boldsymbol{\beta}}+\hat{\boldsymbol{\phi}} v_{d}^{(s)}\right\}\right]\right\}, \\
& \hat{C}_{d}=\frac{1}{2 S} \sum_{s=1}^{2 S} \exp \left\{\hat{\boldsymbol{\phi}} y_{d .} v_{d}^{(s)}-\sum_{i=1}^{n_{d}} m_{d i} \log \left[1+\exp \left\{\boldsymbol{x}_{d i} \hat{\boldsymbol{\beta}}+\hat{\boldsymbol{\phi}} v_{d}^{(s)}\right\}\right]\right\} .
\end{aligned}
$$

Note that the census unit-record data $\left\{\boldsymbol{x}_{d j}\right\}$ is not needed to construct the EBPs (3.3) and (3.4), provided an implicit structure is assumed for the census data and no continuous variables are to be included in the survey-based model. Here we employ a set of marginal tables containing the population sizes $N_{d k}$. If there is no sample, that is $n_{d}=0$ for a given domain $d$, then $\hat{v}_{d}(\hat{\boldsymbol{\theta}})=0$ and $\hat{\mu}_{d}(\hat{\boldsymbol{\theta}})$ can be approximated using a synthetic estimate based on the sampled domains. The method is as follows.

1. As above.

2. Calculate $\hat{\mu}_{d}(\hat{\boldsymbol{\theta}})=\sum_{k=1}^{K} N_{d k} \hat{q}_{d k 0}$, where

$$
\hat{q}_{d k 0}=\frac{1}{2 S} \sum_{s=1}^{2 S}\left\{\frac{\exp \left\{\boldsymbol{z}_{k} \hat{\boldsymbol{\beta}}+\hat{\boldsymbol{\phi}} v_{d}^{(s)}\right\}}{1+\exp \left\{\boldsymbol{z}_{k} \hat{\boldsymbol{\beta}}+\hat{\boldsymbol{\phi}} v_{d}^{(s)}\right\}}\right\} .
$$

The plug-in and the synthetic plug-in estimators of $p_{d j}, \mu_{d}$ and $\bar{\mu}_{d}=\mu_{d} / N_{d}$ do not require running Monte Carlo simulation algorithms. They are

$$
\begin{gathered}
\hat{p}_{d j}^{i n}=\frac{\exp \left\{\boldsymbol{x}_{d j} \hat{\boldsymbol{\beta}}+\hat{v}_{d}\right\}}{1+\exp \left\{\boldsymbol{x}_{d j} \hat{\boldsymbol{\beta}}+\hat{v}_{d}\right\}}, \quad \hat{\mu}_{d}^{i n}=\sum_{k=1}^{K} N_{d k} \frac{\exp \left\{\boldsymbol{z}_{k} \hat{\boldsymbol{\beta}}+\hat{v}_{d}\right\}}{1+\exp \left\{\boldsymbol{z}_{k} \hat{\boldsymbol{\beta}}+\hat{v}_{d}\right\}}, \quad \hat{\bar{\mu}}_{d}^{i n}=\frac{\hat{\bar{\mu}}_{d}^{i n}}{N_{d}}, \\
\hat{p}_{d j}^{s y n}=\frac{\exp \left\{\boldsymbol{x}_{d j} \hat{\boldsymbol{\beta}}\right\}}{1+\exp \left\{\boldsymbol{x}_{d j} \hat{\boldsymbol{\beta}}\right\}}, \quad \hat{\mu}_{d}^{s y n}=\sum_{k=1}^{K} N_{d k} \frac{\exp \left\{\boldsymbol{z}_{k} \hat{\boldsymbol{\beta}}\right\}}{1+\exp \left\{\boldsymbol{z}_{k} \hat{\boldsymbol{\beta}}\right\}}, \quad \hat{\bar{\mu}}_{d}^{s y n}=\frac{\hat{\mu}_{d}^{s y n}}{N_{d}} .
\end{gathered}
$$

Remark 3.1. We can further define the population proportion $\bar{Y}_{d}=\frac{1}{N_{d}} \sum_{j=1}^{N_{d}} y_{d j}$. If $m_{d j}=1$, $d=1, \ldots, D, j=1, \ldots, N_{d}$, it holds that

$$
\begin{aligned}
E\left[\bar{Y}_{d} \mid v_{d}\right] & =\frac{1}{N_{d}} \sum_{j=1}^{N_{d}} p_{d j}=\sum_{k=1}^{K} w_{d k} q_{d k}=\bar{\mu}_{d}, \quad w_{d k}=\frac{N_{d k}}{N_{d}}, \\
\operatorname{var}\left[\bar{Y}_{d} \mid v_{d}\right] & =\frac{1}{N_{d}^{2}} \sum_{j=1}^{N_{d}} p_{d j}\left(1-p_{d j}\right)=\frac{1}{N_{d}} \sum_{k=1}^{K} w_{d k} q_{d k}\left(1-q_{d k}\right)=\frac{\kappa_{d}}{N_{d}}, \quad 0 \leq \kappa_{d} \leq 1 / 4 .
\end{aligned}
$$


By applying the Tchebysheff's inequality, it holds that

$$
P\left(\left|\bar{Y}_{d}-\bar{\mu}_{d}\right|<\varepsilon \mid v_{d}\right) \geq 1-\frac{\operatorname{var}\left[\bar{Y}_{d} \mid v_{d}\right]}{\varepsilon^{2}}=1-\frac{\kappa_{d}}{N_{d} \varepsilon^{2}} .
$$

Equation (3.8) implies, for example, that $P\left(\left|\bar{Y}_{d}-\bar{\mu}_{d}\right|<\varepsilon \mid v_{d}\right) \geq 0.9$ if $\varepsilon=10^{-2}$ and $N_{d}=\kappa_{d} 10^{5}$. Further, if $q_{d k} \approx 0.20$ for all $k$, then $\kappa_{d} \approx 0.16$ and $N_{d} \approx 16,000$ is smaller than the domain sizes appearing in the application to real data presented in Section 6. Thus, $\bar{\mu}_{d}$ can be assumed to be a good approximation of $\bar{Y}_{d}$.

\section{The MSE of the EBP}

This section presents an approximation and gives four estimators of the MSE of the EBP of $\mu_{d}=\mu_{d}\left(\boldsymbol{\theta}, v_{d}\right)$. We assume that $m_{d j}=1, d=1, \ldots, D, j=1, \ldots, n_{d}$, and that all the $n_{d} \mathrm{~s}$ are bounded to be finite. This is the situation of the application to real data of Section 6 . A consequence of the last assumption is that the total sample size $n$ and the number of domains $D$ are of the same order.

The MSE of the EBP can be decomposed into the following form

$$
\begin{aligned}
\operatorname{MSE}\left(\hat{\mu}_{d}\right) & =E\left[\left(\hat{\mu}_{d}(\hat{\boldsymbol{\theta}})-\mu_{d}\left(\boldsymbol{\theta}, v_{d}\right)\right)^{2}\right]=E\left[\left(\left\{\hat{\mu}_{d}(\hat{\boldsymbol{\theta}})-\hat{\mu}_{d}(\boldsymbol{\theta})\right\}+\left\{\hat{\mu}_{d}(\boldsymbol{\theta})-\mu_{d}\left(\boldsymbol{\theta}, v_{d}\right)\right\}\right)^{2}\right] \\
& =E\left[\left(\hat{\mu}_{d}(\hat{\boldsymbol{\theta}})-\hat{\mu}_{d}(\boldsymbol{\theta})\right)^{2}\right]+E\left[\left(\hat{\mu}_{d}(\boldsymbol{\theta})-\mu_{d}\left(\boldsymbol{\theta}, v_{d}\right)\right)^{2}\right] .
\end{aligned}
$$

The second term of $\operatorname{MSE}\left(\hat{\mu}_{d}\right)$ is

$$
\begin{aligned}
g_{d}(\boldsymbol{\theta}) & =E\left[\left(\hat{\mu}_{d}(\boldsymbol{\theta})-\mu_{d}\left(\boldsymbol{\theta}, v_{d}\right)\right)^{2}\right]=E\left[\hat{\mu}_{d}^{2}(\boldsymbol{\theta})\right]+E\left[\mu_{d}^{2}\left(\boldsymbol{\theta}, v_{d}\right)\right]-2 E\left[\hat{\mu}_{d}(\boldsymbol{\theta}) E\left[\mu_{d}\left(\boldsymbol{\theta}, v_{d}\right) \mid \boldsymbol{y}_{d}\right]\right] \\
& =E\left[\mu_{d}^{2}\left(\boldsymbol{\theta}, v_{d}\right)\right]-E\left[\hat{\mu}_{d}^{2}(\boldsymbol{\theta})\right] .
\end{aligned}
$$

The first and second terms of $g_{d}(\boldsymbol{\theta})$ are

$$
\begin{gathered}
E\left[\mu_{d}^{2}\left(\boldsymbol{\theta}, v_{d}\right)\right]=\int_{R}\left(\sum_{k=1}^{K} N_{d k} \frac{\exp \left\{z_{k} \boldsymbol{\beta}+\phi v_{d}\right\}}{1+\exp \left\{\boldsymbol{z}_{k} \boldsymbol{\beta}+\phi v_{d}\right\}}\right)^{2} f\left(v_{d}\right) d v_{d}, \\
E\left[\hat{\mu}_{d}^{2}(\boldsymbol{\theta})\right]=E\left[\psi_{d}^{2}\left(y_{d}, \boldsymbol{\theta}\right)\right]=\sum_{j=0}^{n_{d}} \psi_{d}^{2}(j, \boldsymbol{\theta}) p_{d}(j, \boldsymbol{\theta}),
\end{gathered}
$$

where $\psi_{d}$ was defined in (3.4),

$$
\begin{aligned}
p_{d}(j, \boldsymbol{\theta}) & =P\left(y_{d .}=j\right)=\sum_{\boldsymbol{y}_{d} \in S_{n_{d}, j}} P\left(\boldsymbol{y}_{d}\right)=\sum_{\boldsymbol{y}_{d} \in S_{n_{d}, j}} \int_{R} P\left(\boldsymbol{y}_{d} \mid v_{d}\right) f\left(v_{d}\right) d v_{d} \\
& =\sum_{\boldsymbol{y}_{d} \in S_{n_{d}, j}}\left\{\exp \left\{\sum_{i=1}^{n_{d}} y_{i} \boldsymbol{x}_{d i} \boldsymbol{\beta}\right\} \int_{R} \exp \left\{j \phi v_{d}-\sum_{i=1}^{n_{d}} \log \left[1+\exp \left\{\boldsymbol{x}_{d i} \boldsymbol{\beta}+\boldsymbol{\phi} v_{d}\right\}\right]\right\} f\left(v_{d}\right) d v_{d}\right\}
\end{aligned}
$$

and $S_{n_{d}, j}=\left\{\boldsymbol{y}_{d}=\left(y_{1}, \ldots, y_{n_{d}}\right) \in\{0,1\}^{n_{d}}: y_{d .}=y_{1}+\cdots+y_{n_{d}}=j\right\}$. 
Concerning the first term of $\operatorname{MSE}\left(\hat{\mu}_{d}\right)$, we have

$$
\begin{aligned}
\hat{\mu}_{d}(\hat{\boldsymbol{\theta}})-\hat{\mu}_{d}(\boldsymbol{\theta})= & \psi_{d}\left(y_{d .}, \hat{\boldsymbol{\theta}}\right)-\psi_{d}\left(y_{d .}, \boldsymbol{\theta}\right)=\left(\frac{\partial}{\partial \boldsymbol{\theta}} \psi_{d}\left(y_{d .}, \boldsymbol{\theta}\right)\right)^{\prime}(\hat{\boldsymbol{\theta}}-\boldsymbol{\theta}) \\
& +\frac{1}{2}(\hat{\boldsymbol{\theta}}-\boldsymbol{\theta})^{\prime}\left(\frac{\partial^{2}}{\partial \boldsymbol{\theta}^{2}} \psi_{d}\left(y_{d .}, \boldsymbol{\theta}\right)\right)(\hat{\boldsymbol{\theta}}-\boldsymbol{\theta})+o\left(\|\hat{\boldsymbol{\theta}}-\boldsymbol{\theta}\|^{2}\right) .
\end{aligned}
$$

Hereafter the symbols $o(),. O($.$) are understood in an appropriate sense, for example in$ probability. We assume that the $\boldsymbol{x}_{d j}$ s fulfill the regularity condition (23) of Jiang (2003). Then, we have $|\hat{\boldsymbol{\theta}}-\boldsymbol{\theta}|=O\left(D^{-1 / 2}\right)$ and

$$
E\left[\left(\hat{\mu}_{d}(\hat{\boldsymbol{\theta}})-\hat{\mu}_{d}(\boldsymbol{\theta})\right)^{2}\right]=\frac{1}{D} E\left[\left(\left(\frac{\partial}{\partial \boldsymbol{\theta}} \psi_{d}\left(y_{d .}, \boldsymbol{\theta}\right)\right)^{\prime} \sqrt{D}(\hat{\boldsymbol{\theta}}-\boldsymbol{\theta})\right)^{2}\right]+o(1 / D) .
$$

Now consider $\hat{\boldsymbol{\theta}}_{d-}$, an estimator based on $\boldsymbol{y}_{d-}=\left(\boldsymbol{y}_{d^{\prime}}\right)_{d^{\prime} \neq d}$, and write $\hat{\mu}_{d-}=\psi_{d}\left(y_{d}, \hat{\boldsymbol{\theta}}_{d-}\right)$. By the independence of $\boldsymbol{y}_{d}$ and $\boldsymbol{y}_{d-}$, we have

$$
\begin{aligned}
a_{d}(\boldsymbol{\theta}) & =E\left[\left(\left(\frac{\partial}{\partial \boldsymbol{\theta}} \psi_{d}\left(y_{d .}, \boldsymbol{\theta}\right)\right)^{\prime} \sqrt{D}\left(\hat{\boldsymbol{\theta}}_{d-}-\boldsymbol{\theta}\right)\right)^{2}\right] \\
& =\sum_{j=1}^{n_{d}} E\left[\left(\left(\frac{\partial}{\partial \boldsymbol{\theta}} \psi_{d}\left(y_{d .}, \boldsymbol{\theta}\right)\right)^{\prime} \sqrt{D}\left(\hat{\boldsymbol{\theta}}_{d-}-\boldsymbol{\theta}\right)\right)^{2} \mid y_{d .}=j\right] p_{d}(j, \boldsymbol{\theta}) \\
& =\sum_{j=1}^{n_{d}}\left(\frac{\partial}{\partial \boldsymbol{\theta}} \psi_{d}(j, \boldsymbol{\theta})\right)^{\prime} \boldsymbol{V}_{d}(\boldsymbol{\theta})\left(\frac{\partial}{\partial \boldsymbol{\theta}} \psi_{d}(j, \boldsymbol{\theta})\right) p_{d}(j, \boldsymbol{\theta}),
\end{aligned}
$$

where $\boldsymbol{V}_{d}(\boldsymbol{\theta})=D E\left[\left(\hat{\boldsymbol{\theta}}_{d-}-\boldsymbol{\theta}\right)\left(\hat{\boldsymbol{\theta}}_{d-}-\boldsymbol{\theta}\right)^{\prime} \mid y_{d .}=j\right]=D E\left[\left(\hat{\boldsymbol{\theta}}_{d-}-\boldsymbol{\theta}\right)\left(\hat{\boldsymbol{\theta}}_{d_{-}}-\boldsymbol{\theta}\right)^{\prime}\right]$. Therefore,

$$
\operatorname{MSE}\left(\hat{\mu}_{d^{-}}\right)=g_{d}(\boldsymbol{\theta})+\frac{1}{D} a_{d}(\boldsymbol{\theta})+o(1 / D) .
$$

If the $\boldsymbol{x}_{d j}$ S also fulfill the regularity conditions (24) and (25) of Jiang (2003), then we may replace $\hat{\boldsymbol{\theta}}_{d-}$ by $\hat{\boldsymbol{\theta}}$, an estimator of $\boldsymbol{\theta}$ based on all data, and we obtain

$$
\operatorname{MSE}\left(\hat{\mu}_{d}\right)=g_{d}(\boldsymbol{\theta})+\frac{1}{D} c_{d}(\boldsymbol{\theta})+o(1 / D)
$$

where

$$
c_{d}(\boldsymbol{\theta})=\sum_{j=1}^{n_{d}}\left(\frac{\partial}{\partial \boldsymbol{\theta}} \psi_{d}(j, \boldsymbol{\theta})\right)^{\prime} \boldsymbol{V}(\boldsymbol{\theta})\left(\frac{\partial}{\partial \boldsymbol{\theta}} \psi_{d}(j, \boldsymbol{\theta})\right) p_{d}(j, \boldsymbol{\theta}), \quad \boldsymbol{V}(\boldsymbol{\theta})=D E\left[(\hat{\boldsymbol{\theta}}-\boldsymbol{\theta})(\hat{\boldsymbol{\theta}}-\boldsymbol{\theta})^{\prime}\right] .
$$

A plug-in estimator of $\operatorname{MSE}\left(\hat{\mu}_{d}\right)$ is

$$
m s e^{P}\left(\hat{\mu}_{d}\right)=g_{d}(\hat{\boldsymbol{\theta}})+\frac{1}{D} c_{d}(\hat{\boldsymbol{\theta}}) .
$$

We have that $E\left[c_{d}(\hat{\boldsymbol{\theta}})-c_{d}(\boldsymbol{\theta})\right]=o(1)$. However $E\left[g_{d}(\hat{\boldsymbol{\theta}})-g_{d}(\boldsymbol{\theta})\right]$ is not of order $o\left(D^{-1}\right)$. 
Let $\hat{\boldsymbol{\theta}}$ be a truncated MM estimator. This is to say

$$
\hat{\beta}_{k}= \begin{cases}-L_{n} & \text { if } \tilde{\beta}_{k}<-L_{n} \\
\tilde{\beta}_{k} & \text { if }-L_{n}<\tilde{\beta}_{k}<L_{n}, \quad \hat{\phi}=\left\{\begin{array}{ll}
\tilde{\phi} & \text { if } \tilde{\phi} \leq L_{n} \\
L_{n} & \text { if } \tilde{\beta}_{k}>L_{n}
\end{array} \text { if } \tilde{\phi}>L_{n}\right.\end{cases}
$$

where $\tilde{\boldsymbol{\theta}}=\left(\tilde{\beta}_{1}, \ldots, \tilde{\beta}_{p}, \tilde{\phi}\right)^{\prime}$ is an MM estimator. Under regularity conditions (23)-(25) of Jiang (2003) it can be proved that $E[\hat{\boldsymbol{\theta}}-\boldsymbol{\theta}]=O\left(D^{-1}\right)$ holds for the MM and for the truncated MM estimator (see Jiang 2003, 123). In what follows, we assume that $E[\hat{\boldsymbol{\theta}}-\boldsymbol{\theta}]=O\left(D^{-1}\right)$ holds. By the Taylor expansion, we have

$$
g_{d}(\hat{\boldsymbol{\theta}})=g_{d}(\boldsymbol{\theta})+\left(\frac{\partial}{\partial \boldsymbol{\theta}} g_{d}(\boldsymbol{\theta})\right)^{\prime}(\hat{\boldsymbol{\theta}}-\boldsymbol{\theta})+\frac{1}{2}(\hat{\boldsymbol{\theta}}-\boldsymbol{\theta})^{\prime}\left(\frac{\partial^{2}}{\partial \boldsymbol{\theta}^{2}} g_{d}(\boldsymbol{\theta})\right)(\hat{\boldsymbol{\theta}}-\boldsymbol{\theta})+o\left(D^{-1}\right),
$$

and hence

$$
E\left[g_{d}(\hat{\boldsymbol{\theta}})\right]=g_{d}(\boldsymbol{\theta})+\frac{1}{D} b_{d}(\boldsymbol{\theta})+o\left(D^{-1}\right)
$$

where

$$
b_{d}(\boldsymbol{\theta})=\left(\frac{\partial}{\partial \boldsymbol{\theta}} g_{d}(\boldsymbol{\theta})\right)^{\prime} D E[\hat{\boldsymbol{\theta}}-\boldsymbol{\theta}]+\frac{1}{2} E\left[\sqrt{D}(\hat{\boldsymbol{\theta}}-\boldsymbol{\theta})^{\prime}\left(\frac{\partial^{2}}{\partial \boldsymbol{\theta}^{2}} g_{d}(\boldsymbol{\theta})\right) \sqrt{D}(\hat{\boldsymbol{\theta}}-\boldsymbol{\theta})\right] .
$$

Proposition 4.1 gives an approximation to the bias term $b_{d}$ when $\hat{\boldsymbol{\theta}}$ is the truncated MM estimator. Appendix A.2 gives the proof.

Proposition 4.1. Let $\hat{\boldsymbol{\theta}}$ be the truncated MM estimator. Under regularity conditions (23)-(25) of Jiang (2003), it holds that $b_{d}(\boldsymbol{\theta})=B_{d}(\boldsymbol{\theta})+o(1)$, where

$$
\begin{aligned}
B_{d}(\boldsymbol{\theta}) & =\frac{1}{2}\left\{E\left[r_{D, d}\right]-\left(\frac{\partial}{\partial \boldsymbol{\theta}} g_{d}(\boldsymbol{\theta})\right)^{\prime}\left(\frac{\partial}{\partial \boldsymbol{\theta}} \boldsymbol{M}(\boldsymbol{\theta})\right)^{-1} E\left[\boldsymbol{q}_{D}\right]\right\} \\
r_{D, d} & =\Delta_{D}^{\prime} \boldsymbol{R}_{d}(\boldsymbol{\theta}) \Delta_{D}, \quad \boldsymbol{R}_{d}(\boldsymbol{\theta})=\left(\left(\frac{\partial}{\partial \boldsymbol{\theta}} \boldsymbol{M}(\boldsymbol{\theta})\right)^{-1}\right)^{\prime}\left(\frac{\partial^{2}}{\partial \boldsymbol{\theta}^{2}} g_{d}(\boldsymbol{\theta})\right)\left(\frac{\partial}{\partial \boldsymbol{\theta}} \boldsymbol{M}(\boldsymbol{\theta})\right)^{-1}, \\
\boldsymbol{q}_{D} & ={ }_{1 \leq k \leq p+1}^{\operatorname{col}}\left(q_{D k}\right), \quad \boldsymbol{M}(\boldsymbol{\theta})=\underset{1 \leq k \leq p+1}{\operatorname{col}}\left(M_{k}(\boldsymbol{\theta})\right), \quad \hat{\boldsymbol{M}}=\underset{1 \leq k \leq p+1}{\operatorname{col}}\left(\hat{M}_{k}\right), \\
q_{D k} & =\Delta_{D}^{\prime} \boldsymbol{Q}_{k}(\boldsymbol{\theta}) \Delta_{D}, \quad \boldsymbol{Q}_{k}(\boldsymbol{\theta})=\left(\left(\frac{\partial}{\partial \boldsymbol{\theta}} \boldsymbol{M}(\boldsymbol{\theta})\right)^{-1}\right)^{\prime}\left(\frac{\partial^{2}}{\partial \boldsymbol{\theta}^{2}} M_{k}(\boldsymbol{\theta})\right)\left(\frac{\partial}{\partial \theta} \boldsymbol{M}(\boldsymbol{\theta})\right)^{-1}, \\
\Delta_{D} & =\sqrt{D}(\hat{\boldsymbol{M}}-\boldsymbol{M}(\boldsymbol{\theta})), \quad \frac{\partial}{\partial \boldsymbol{\theta}} \boldsymbol{M}(\boldsymbol{\theta})=\left(\frac{\partial}{\partial \boldsymbol{\theta}_{k_{2}}} \boldsymbol{M}_{k_{1}}(\boldsymbol{\theta})\right)_{k_{1}, k_{2}=1, \ldots, p+1}
\end{aligned}
$$


Finally, an order $o\left(D^{-1}\right)$ theoretical estimator of the MSE with bias correction is

$$
\widehat{M S E}\left(\hat{\mu}_{d}\right)=g_{d}(\hat{\boldsymbol{\theta}})+\frac{1}{D} c_{d}(\hat{\boldsymbol{\theta}})-\frac{1}{D} B_{d}(\hat{\boldsymbol{\theta}})
$$

and the practical estimators, without and with bias correction, are

$$
m s e^{0}\left(\hat{\mu}_{d}\right)=\hat{g}_{d}(\hat{\boldsymbol{\theta}})+\frac{1}{D} \hat{c}_{d}(\hat{\boldsymbol{\theta}}), \quad m s e^{1}\left(\hat{\mu}_{d}\right)=m s e^{0}\left(\hat{\mu}_{d}\right)-\frac{1}{D} \hat{B}_{d}(\hat{\boldsymbol{\theta}})
$$

Appendix A.3 gives the Monte Carlo approximations $\hat{g}_{d}(\hat{\boldsymbol{\theta}})$ and $\hat{c}_{d}(\hat{\boldsymbol{\theta}})$ of $g_{d}(\hat{\boldsymbol{\theta}})$ and $c_{d}(\hat{\boldsymbol{\theta}})$ respectively, and the bootstrap estimator $\hat{B}_{d}(\hat{\boldsymbol{\theta}})$ of $B_{d}(\boldsymbol{\theta})$. Appendix A.4 presents formulas of some derivatives needed to evaluate the abovementioned approximations.

Another approach to estimating the MSE is to use a parametric bootstrap. The following procedure calculates a bootstrap and a double-bootstrap bias-corrected estimator of $\operatorname{MSE}\left(\hat{\mu}_{d}\right)$.

1. Fit the model to the sample and calculate $\hat{\boldsymbol{\theta}}=\left(\hat{\beta}^{\prime}, \hat{\phi}\right)^{\prime}$.

2. Repeat $B_{1}$ times $\left(b_{1}=1, \ldots, B_{1}\right)$ :

(a) For $d=1, \ldots, D, j=1, \ldots, n_{d}$, generate $v_{d}^{*\left(b_{1}\right)}$ i.i.d. $\mathrm{N}(0,1)$ and calculate

$$
\begin{gathered}
p_{d j}^{*\left(b_{1}\right)}=\frac{\exp \left\{\boldsymbol{x}_{d j} \hat{\boldsymbol{\beta}}+\hat{\boldsymbol{\phi}} v_{d}^{*\left(b_{1}\right)}\right\}}{1+\exp \left\{\boldsymbol{x}_{d j} \hat{\boldsymbol{\beta}}+\hat{\boldsymbol{\phi}} v_{d}^{*\left(b_{1}\right)}\right\}}, \quad y_{d j}^{*\left(b_{1}\right)} \sim \operatorname{Bin}\left(m_{d j}, p_{d j}^{*\left(b_{1}\right)}\right), \\
\mu_{d}^{*\left(b_{1}\right)}=\mu_{d}\left(\hat{\boldsymbol{\theta}}, v_{d}^{*\left(b_{1}\right)}\right)=\sum_{k=1}^{K} N_{d k} q_{d k}^{*\left(b_{1}\right)}, \quad q_{d k}^{*\left(b_{1}\right)}=\frac{\exp \left\{\boldsymbol{z}_{k} \hat{\boldsymbol{\beta}}+\hat{\boldsymbol{\phi}} v_{d}^{*\left(b_{1}\right)}\right\}}{1+\exp \left\{\boldsymbol{z}_{k} \hat{\boldsymbol{\beta}}+\hat{\boldsymbol{\phi}} v_{d}^{*\left(b_{1}\right)}\right\}} .
\end{gathered}
$$

(b) For each bootstrap sample, calculate $\hat{\boldsymbol{\theta}}^{*\left(b_{1}\right)}$ and the EBP $\hat{\mu}_{d}^{*\left(b_{1}\right)}=\hat{\mu}_{d}\left(\hat{\boldsymbol{\theta}}^{*\left(b_{1}\right)}\right)$.

(c) Repeat $B_{2}$ times $\left(b_{2}=1, \ldots, B_{2}\right)$ :

i. For $d=1, \ldots, D, j=1, \ldots, n_{d}$, generate $v_{d}^{*\left(b_{1}, b_{2}\right)}$ i.i.d. $\mathrm{N}(0,1)$ and calculate

$$
\begin{aligned}
& p_{d j}^{*\left(b_{1}, b_{2}\right)}= \frac{\exp \left\{\boldsymbol{x}_{d j} \hat{\boldsymbol{\beta}}^{*\left(b_{1}\right)}+\hat{\boldsymbol{\phi}}^{*\left(b_{1}\right)} v_{d}^{*\left(b_{1}, b_{2}\right)}\right\}}{1+\exp \left\{\boldsymbol{x}_{d j} \hat{\boldsymbol{\beta}}^{*\left(b_{1}\right)}+\hat{\boldsymbol{\phi}}^{*\left(b_{1}\right)} v_{d}^{*\left(b_{1}, b_{2}\right)}\right\}}, \\
& y_{d j}^{*\left(b_{1}, b_{2}\right)} \sim \operatorname{Bin}\left(m_{d j}, p_{d j}^{*\left(b_{1}, b_{2}\right)}\right), \\
& \mu_{d}^{*\left(b_{1}, b_{2}\right)}=\mu_{d}\left(\hat{\boldsymbol{\theta}}^{*\left(b_{1}\right)}, v_{d}^{*\left(b_{1}, b_{2}\right)}\right)=\sum_{k=1}^{K} N_{d k} q_{d k}^{*\left(b_{1}, b_{2}\right)}, \\
& q_{d k}^{*\left(b_{1}, b_{2}\right)}=\frac{\exp \left\{\boldsymbol{z}_{k} \hat{\boldsymbol{\beta}}^{*\left(b_{1}\right)}+\hat{\boldsymbol{\phi}}^{*\left(b_{1}\right)} v_{d}^{*\left(b_{1}, b_{2}\right)}\right\}}{1+\exp \left\{\boldsymbol{z}_{k} \hat{\boldsymbol{\beta}}^{*\left(b_{1}\right)}+\hat{\boldsymbol{\phi}}^{*\left(b_{1}\right)} v_{d}^{*\left(b_{1}, b_{2}\right)}\right\}} .
\end{aligned}
$$

ii. For each bootstrap sample, calculate $\hat{\boldsymbol{\theta}}^{*\left(b_{1}, b_{2}\right)}$ and the EBP $\hat{\mu}_{d}^{*\left(b_{1}, b_{2}\right)}=\hat{\mu}_{d}\left(\hat{\boldsymbol{\theta}}^{*\left(b_{1}, b_{2}\right)}\right)$.

iii. Output: $\operatorname{mse}_{d}^{*\left(b_{1}\right)}=\frac{1}{B_{2}} \sum_{b_{2}=1}^{B_{2}}\left(\hat{\mu}_{d}^{*\left(b_{1}, b_{2}\right)}-\mu_{d}^{*\left(b_{1}, b_{2}\right)}\right)^{2}$. 
3. Output:

$$
m s e^{*}\left(\hat{\mu}_{d}\right)=\frac{1}{B_{1}} \sum_{b_{1}=1}^{B_{1}}\left(\hat{\mu}_{d}^{*\left(b_{1}\right)}-\mu_{d}^{*\left(b_{1}\right)}\right)^{2}, \quad m s e^{* *}\left(\hat{\mu}_{d}\right)=2 m s e^{*}\left(\hat{\mu}_{d}\right)-\frac{1}{B_{1}} \sum_{b_{1}=1}^{B_{1}} m s e_{d}^{*\left(b_{1}\right)} .
$$

\section{Simulation Experiments}

In this section we present three simulation experiments. They are fully model-based and are linked to the case study of Section 6, but with much smaller sample and population sizes. All of them use the same simulation environment, which can be described in the following way. Take $N_{d}=1,000, n_{d}=5,10,20,40, D=30$. Note that, to enable computation, $N_{d}$ is more than an order of magnitude smaller than specified in the approximation in probability used in (3.8). For $d=1, \ldots, D, j=1, \ldots, n_{d}$, generate regressors, $x_{d j 1}$ and $x_{d j 2}$, classifying individuals into one of three possible classes (e.g., inactive, unemployed, and employed), so that they take on values $\left(x_{d j 1}, x_{d j 2}\right) \in$ $\{(0,0),(0,1),(1,0)\}$ with probabilities $0.3,0.2$, and 0.5 , respectively. Generate $v_{d} \sim N(0,1), d=1, \ldots, D$. Take $\boldsymbol{\beta}=\left(\beta_{0}, \beta_{1}, \beta_{2}\right)=(1 / 3,-3 / 2,1 / 2)$ and $\phi=1 / 2$. For $d=1, \ldots, D, j=1, \ldots, n_{d}$, generate the target variable

$$
y_{d j} \sim \operatorname{Bin}\left(m_{d j}, p_{d j}\right), \quad p_{d j}=\frac{\exp \left\{\beta_{0}+x_{d j 1} \beta_{1}+x_{d j 2} \beta_{2}+\phi v_{d}\right\}}{1+\exp \left\{\beta_{0}+x_{d j 1} \beta_{1}+x_{d j 2} \beta_{2}+\phi v_{d}\right\}}, \quad m_{d j}=1,
$$

where $y_{d j}=1(=0)$ indicates that individual $j$ of domain $d$ is (not) below the poverty line and $p_{d j}$ is the corresponding binomial probability. We choose $D=30$ as a round figure close to the number $D=34$ of domains in the real data.

As some of the theoretical results are asymptotic, we investigate cases with small to medium sample sizes. The selected scenario resembles the application to real data. For computational reasons, the population size $N=30,000$ is much smaller than the population size $N=4,990.277$ of the study case. Nevertheless, the simulations are illustrative and give useful information about how the methodology works in practice. Simulation 1 investigates the behavior of the model parameter estimators. Simulation 2 calculates the bias and the MSE of the EBP and the plug-in estimators under different scenarios. Simulation 3 compares the introduced MSE estimators.

\subsection{Simulation 1}

The target of Simulation 1 is to check the behavior of the fitting algorithm. The steps of Simulation 1 are

1. Repeat $\mathrm{K}=1,000$ times $(k=1, \ldots, K)$.

1.1. Generate a sample of size $n=\sum_{d=1}^{D} n_{d}$. Calculate $\hat{\beta}_{0}^{(k)}, \hat{\beta}_{1}^{(k)}, \hat{\beta}_{2}^{(k)}$ and $\hat{\phi}^{(k)}$.

2. Output: For $\theta \in\left\{\beta_{0}, \beta_{1}, \beta_{2}, \phi\right\}$, calculate the empirical bias and the root-MSE

$$
B I A S=\frac{1}{K} \sum_{k=1}^{K}\left(\hat{\theta}^{(k)}-\theta\right), \quad R M S E=\left(\frac{1}{K} \sum_{k=1}^{K}\left(\hat{\theta}^{(k)}-\theta\right)^{2}\right)^{1 / 2}
$$


Table 1. BIAS (left) and RMSE (right) for $D=30$.

\begin{tabular}{lrrrr|rrrr}
\hline$n$ & 150 & 300 & 600 & 1,200 & 150 & 300 & 600 & 1,200 \\
$n_{d}$ & 5 & 10 & 20 & 40 & 5 & 10 & 20 & 40 \\
\hline$\hat{\beta}_{0}$ & 0.004 & -0.004 & -0.003 & -0.001 & 0.274 & 0.242 & 0.180 & 0.156 \\
$\hat{\beta}_{1}$ & -0.002 & -0.005 & 0.006 & 0.004 & 0.346 & 0.301 & 0.223 & 0.158 \\
$\hat{\beta}_{2}$ & -0.012 & 0.010 & 0.010 & 0.007 & 0.474 & 0.372 & 0.261 & 0.187 \\
$\hat{\phi}$ & -0.103 & -0.058 & -0.042 & -0.022 & 0.359 & 0.270 & 0.177 & 0.116 \\
\hline
\end{tabular}

Table 1 presents the obtained results. This table shows that the empirical bias and root-MSE of the MSM estimators of the model parameters decrease as the sample size increases. This is coherent with the consistency property given by Theorem 1 of Jiang (1998).

\subsection{Simulation 2}

The target of Simulation 2 is to investigate the behavior of the EBP, the plug-in (IN) and synthetic plug-in estimators (SYN). The steps of Simulation 2 are

1. Repeat $K=10,000$ times $(k=1, \ldots, K)$

1.1. Generate the population in the same way as described at the beginning of this section and calculate

$$
\bar{\mu}_{d}^{(k)}=\frac{1}{N_{d}} \sum_{j=1}^{N_{d}} p_{d j}^{(k)}, \quad \bar{Y}_{d}^{(k)}=\frac{1}{N_{d}} \sum_{j=1}^{N_{d}} y_{d j}^{(k)}, \quad \nabla_{d}^{(k)}=\bar{\mu}_{d}^{(k)}-\bar{Y}_{d}^{(k)} .
$$

1.2. For $d=1, \ldots, D$, select a simple random sample $s_{d}$ (without replacement) of size $n_{d}$ and calculate $\hat{\boldsymbol{\theta}}^{(k)}=\left(\hat{\beta}_{0}^{(k)}, \hat{\boldsymbol{\beta}}_{1}^{(k)}, \hat{\beta}_{2}^{(k)}, \hat{\phi}^{(k)}\right)$ and $\tilde{\boldsymbol{\theta}}^{(k)}=\left(\tilde{\beta}_{0}^{(k)}, \tilde{\beta}_{1}^{(k)}, \tilde{\boldsymbol{\beta}}_{2}^{(k)}\right)$ under the logit model with and without random effects.

1.3. Calculate $\hat{v}_{d}^{(k)}=\hat{v}_{d}\left(\hat{\boldsymbol{\theta}}^{(k)}\right)$ and

$$
\hat{\mu}_{d}^{e b p, k}=\frac{\hat{\mu}_{d}\left(\hat{\boldsymbol{\theta}}^{(k)}\right)}{N_{d}}, \quad \hat{\mu}_{d}^{i n, k}=\frac{\hat{\mu}_{d}^{i n}\left(\hat{\boldsymbol{\theta}}^{(k)}, \hat{v}_{d}^{(k)}\right)}{N_{d}}, \quad \hat{\mu}_{d}^{s y n, k}=\frac{\hat{\mu}_{d}^{s y n}\left(\tilde{\boldsymbol{\theta}}^{(k)}\right)}{N_{d}}, \quad d=1, \ldots, D .
$$

2. For each $\hat{\bar{\mu}}_{d} \in\left\{\hat{\bar{\mu}}_{d}^{e b p}, \hat{\bar{\mu}}_{d}^{i n}, \hat{\bar{\mu}}_{d}^{s y n}\right\}$, calculate

$$
B_{d}=\frac{1}{K} \sum_{k=1}^{K}\left(\hat{\bar{\mu}}_{d}^{k}-\bar{\mu}_{d}^{(k)}\right), \quad E_{d}=\frac{1}{K} \sum_{k=1}^{K}\left(\hat{\bar{\mu}}_{d}^{k}-\bar{\mu}_{d}^{(k)}\right)^{2}, \quad d=1, \ldots, D .
$$

Figures 1, 2, and 3 present the boxplots of the empirical biases, $B_{d} \mathrm{~s}$, and mean-square errors, $E_{d} \mathrm{~s}$, of the EBP, the IN and the SYN defined in (3.4), (3.6), and (3.7) respectively. The SYN is based on the model without random effects and it is calculated from the corresponding parameter estimates $\tilde{\boldsymbol{\theta}}$. Figures 1 and 3 show that the MSE of the EBP is lower than the MSE of the SYN, overall for domain samples sizes greater than ten. Furthermore, the EBP has slightly lower bias than the SYN. Further, the EBP has slightly lower bias and MSE than the IN. 

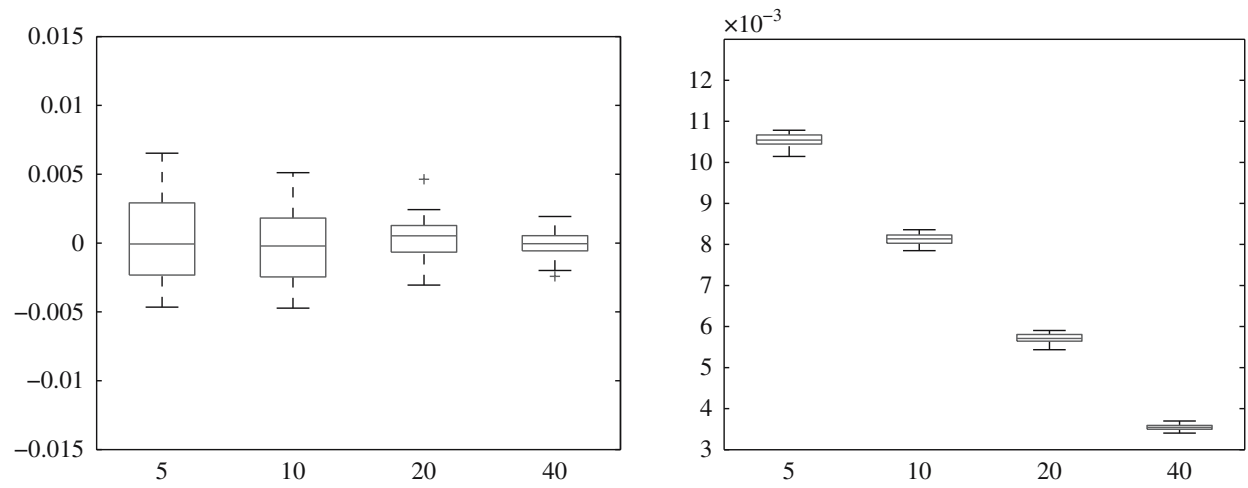

Fig. 1. Boxplots of $B_{d}$ (left) and $E_{d}$ (right) defined in (5.1) for EBP and $n_{d} \in\{5,10,20,40\}$.

Let us illustrate the difference between domain parameters $\bar{\mu}_{d}^{(k)}$ and $\bar{Y}_{d}^{(k)}$ in the simulations. As the calculated differences do not depend on $d$ in the simulated scenario, Table 2 presents the quantiles of $\nabla_{d}^{(k)}$ for $N_{d}=1,000$ and $d=1$. In the interquartile range, the absolute difference is lower than $10^{-2}$.

\subsection{Simulation 3}

The target of Simulation 3 is to investigate the behavior of the four MSE estimators, the analytic estimator $m s e^{0}\left(\hat{\bar{\mu}}_{d}\right)$, the analytic estimator with bias correction $m s e^{1}\left(\hat{\bar{\mu}}_{d}\right)$, the bootstrap estimator $m s e^{*}\left(\hat{\overline{\mu_{d}}}\right)$ and the double-bootstrap bias-corrected estimator $m s e^{* *}\left(\hat{\overline{\mu_{d}}}\right)$ of the EBP. The number of first-stage bootstrap resamples is $B_{1}=100$. By following Erciulescu and Fuller (2014), the number of second-stage bootstrap resamples is $B_{2}=1$. The steps of Simulation 3 are

1. Repeat $K=1,000$ times $(k=1, \ldots, K)$

1.1. Generate the population in the same way as described at the beginning of this section.

1.2. For $d=1, \ldots, D$, select a simple random sample $s_{d}$ (without replacement) of size $n_{d}$. Calculate $m s e_{d}^{0(k)}=m s e^{0}\left(\hat{\mu}_{d}\right) / N_{d}^{2}, m s e_{d}^{1(k)}=m s e^{1}\left(\hat{\mu}_{d}\right) / N_{d}^{2}, m s e_{d}^{*(k)}=$ $m s e^{*}\left(\hat{\mu}_{d}\right) / N_{d}^{2}$ and $m s e_{d}^{* *(k)}=m s e^{* *}\left(\hat{\mu}_{d}\right) / N_{d}^{2}, d=1, \ldots, D$.
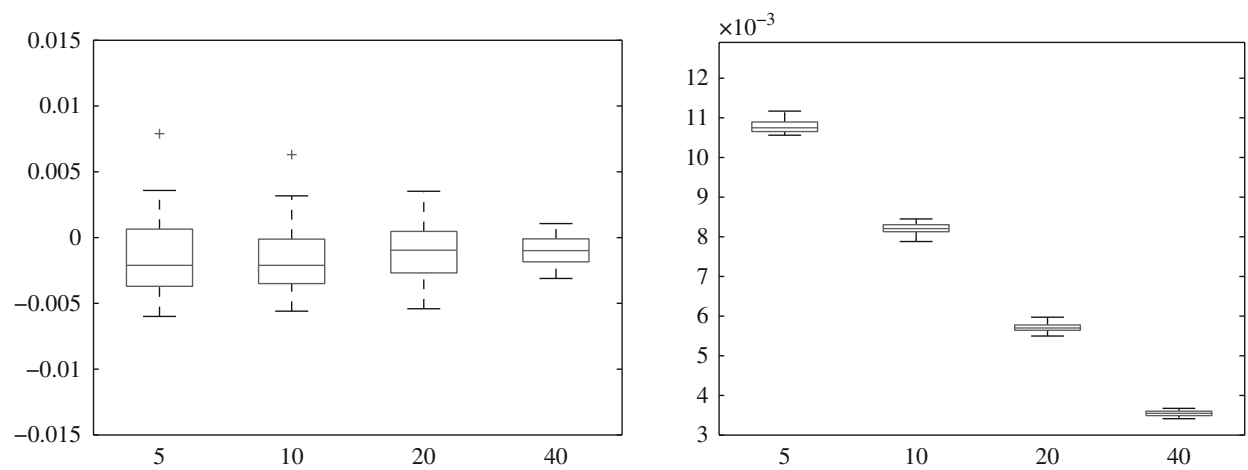

Fig. 2. Boxplots of $B_{d}$ (left) and $E_{d}$ (right) defined in (5.1) for $I N$ and $n_{d} \in\{5,10,20,40\}$. 

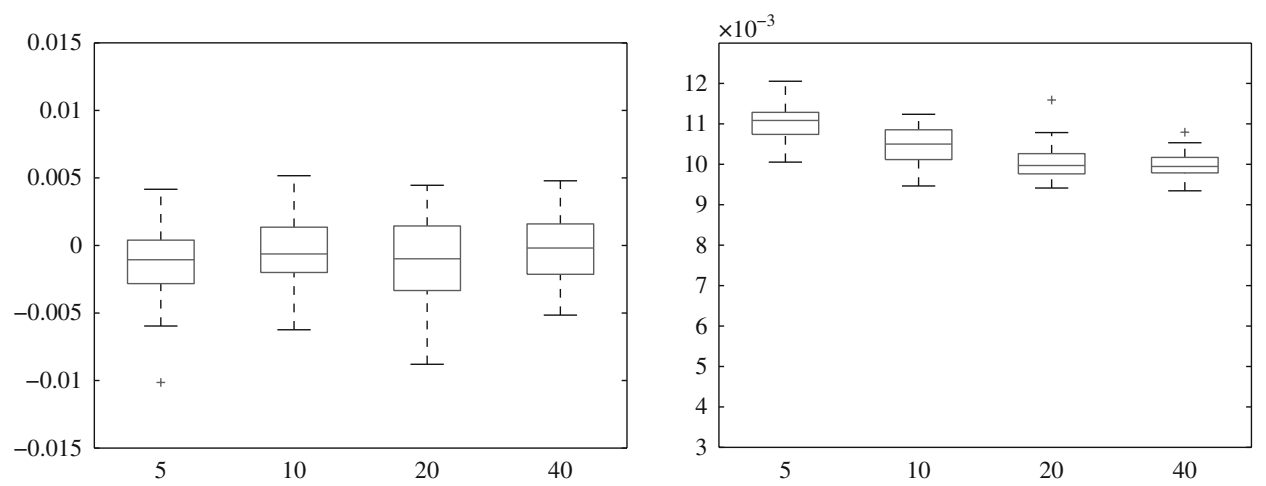

Fig. 3. Boxplots of $B_{d}$ (left) and $E_{d}$ (right) defined in (5.1) for SYN and $n_{d} \in\{5,10,20,40\}$.

2. For every $m s e \in\left\{m s e^{0}, m s e^{1}, m s e^{*}, m s e^{* *}\right\}$, calculate

$$
m s e_{d}=\frac{1}{K} \sum_{k=1}^{K} m s e_{d}^{(k)}, \quad e_{d}=\frac{1}{K} \sum_{k=1}^{K}\left(m s e_{d}^{(k)}-E_{d}^{e b p}\right)^{2}, \quad d=1, \ldots, D,
$$

where $E_{d}^{e b p}, d=1, \ldots, D$, is taken from the output of Simulation 2 .

Figure 4 presents the plots of the four MSE estimators. This figure shows that the bias correction used in $m s e_{d}^{1}$ as well as in $m s e_{d}^{* *}$ does not work for domain sample sizes around $n_{d}=10$. It starts to be effective if $n_{d} \geq 20$, when both $m s e_{d}^{1}$ and $m s e_{d}^{* *}$ seem to estimate the real value of the simulated mean-squared error $E_{d}^{e b p}$ quite well.

Figure 5 contains the boxplots of the empirical MSEs, $e_{d}^{0}, e_{d}^{1}, e_{d}^{*}$ and $e_{d}^{* *}$, of the MSE estimators $m s e_{d}^{0}, m s e_{d}^{1}, m s e_{d}^{*}$ and $m s e_{d}^{* *}$. The analytic estimators, $m s e_{d}^{0}$ and $m s e_{d}^{1}$, perform better than the parametric bootstrap estimators $m s e_{d}^{*}, m s e_{d}^{* *}$. Further, the bias-corrected estimator, $m s e_{d}^{1}$, has the lowest MSEs. It can be also seen that in the case of the doublebootstrap estimator $m s e_{d}^{* *}$ the gained bias correction comes at the cost of increased variability of this estimator.

Remark 5.1. The approximation of terms $g_{d}, c_{d}$ and $B_{d}$ used correspondingly in the $m s e_{d}^{0}$ and $m s e_{d}^{1}$ estimators entails some implementational and computational difficulties. First of all, to be able to calculate the $B_{d}$ term one has to implement all formulas for the needed derivatives, which are partly presented in Appendix A.4. A greater difficulty is that the calculation of the probabilities (4.1) and their derivatives appearing in the $g_{d}, c_{d}$ and $B_{d}$ terms is computationally expensive if $n_{d} \geq 40$, because calculating the sum in (4.1) requires an iterative enumeration of all the elements of the subset $S_{n_{d}, j} \subset\{0,1\}^{n_{d}}$ and the size of $S_{n_{d}, j}$ is $\left(\begin{array}{l}n_{d} \\ j\end{array}\right)$, which increases exponentially with $n_{d}$ when $j$ is around $n_{d} / 2$, for

Table 2. Quantiles of $\nabla_{d}^{(k)}$ for $N_{d}=1,000, d=1$.

\begin{tabular}{lccccr}
\hline$\nabla_{1}$ & $\min$ & $Q_{1}$ & $Q_{2}$ & $Q_{3}$ & $\max$ \\
\hline Quantiles & -0.0549 & -0.0098 & -0.0003 & 0.0093 & 0.0559 \\
\hline
\end{tabular}



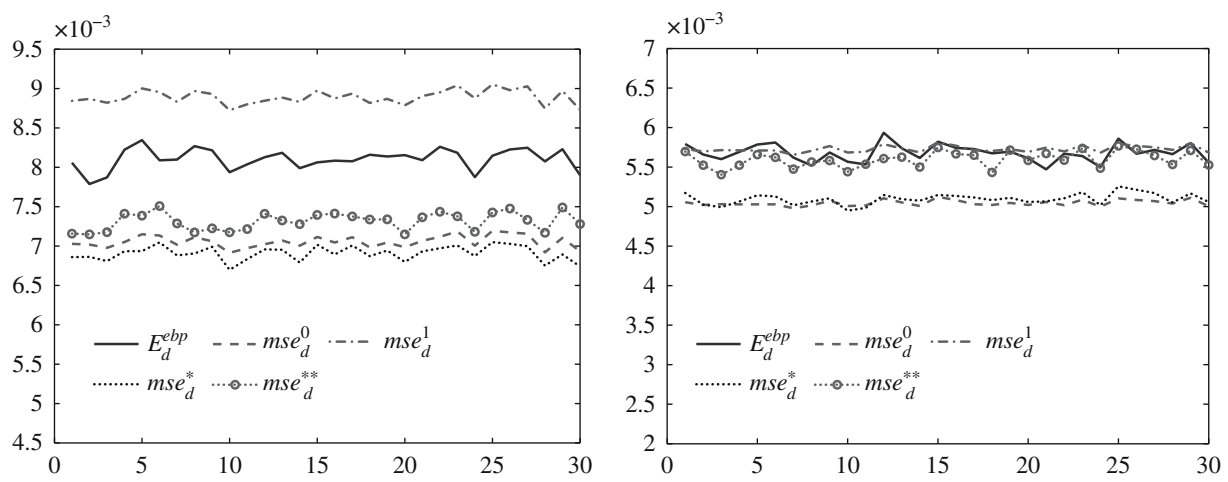

Fig. 4. Plots of $E_{d}^{e b p}, m s e_{d}^{0}$, $m s e_{d}^{l}$ and $m s e_{d}^{*}$ for $n_{d}=10$ (left) and $n_{d}=20$ (right).

example if $n_{d}=40$ the sum over the set $S_{40,20}$ has around $1.4 \cdot 10^{11}$ summands which must be evaluated and counted up for each domain $d$. This difficulty can be solved in some manner in a simulation experiment when the sizes $n_{d}$ are small and the same for all $d$ so that the set of all possible $\boldsymbol{y}_{d} \in S_{n_{d}, j}$ can be stored once in memory or on a disc. But in practical application when the sizes $n_{d}$ are different and some of them are large, there are complications with implementation and computation time.

The parametric bootstrap MSE estimators, $m s e_{d}^{*}, m s e_{d}^{* *}$, avoid the computational problems of their analytic counterparts, $m s e_{d}^{0}$ and $m s e_{d}^{1}$, and present quite good behavior. To illustrate the performance of the bootstrap estimator $m s e_{d}^{*}$ for higher values of $n_{d}$, in Figure 6 we present results of this estimator for $n_{d}=40$. We can observe that for this sample size, the estimator $m s e_{d}^{*}$ is practically unbiased and the double bootstrap is not needed.

\section{Application to SLCS Data}

Alleviating poverty is one of the main social tasks in the European Union (EU). Following the instructions of EUROSTAT, European countries implement a Living Conditions
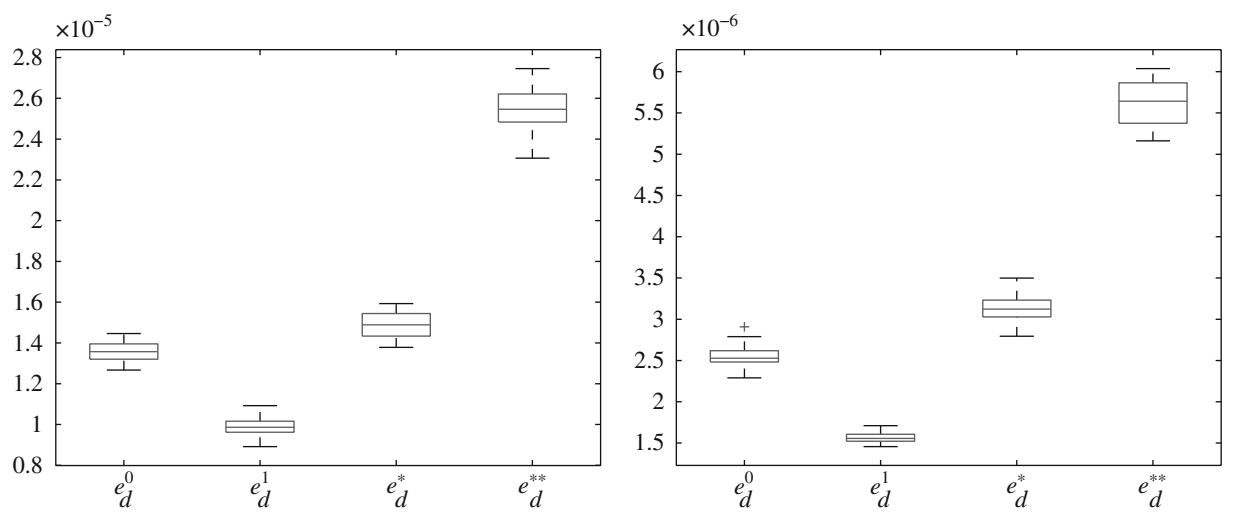

Fig. 5. Plots of $e_{d}^{0}$, $e_{d}^{l}$ and $e_{d}^{*}$ for $n_{d}=10$ (left) and $n_{d}=20$ (right). 


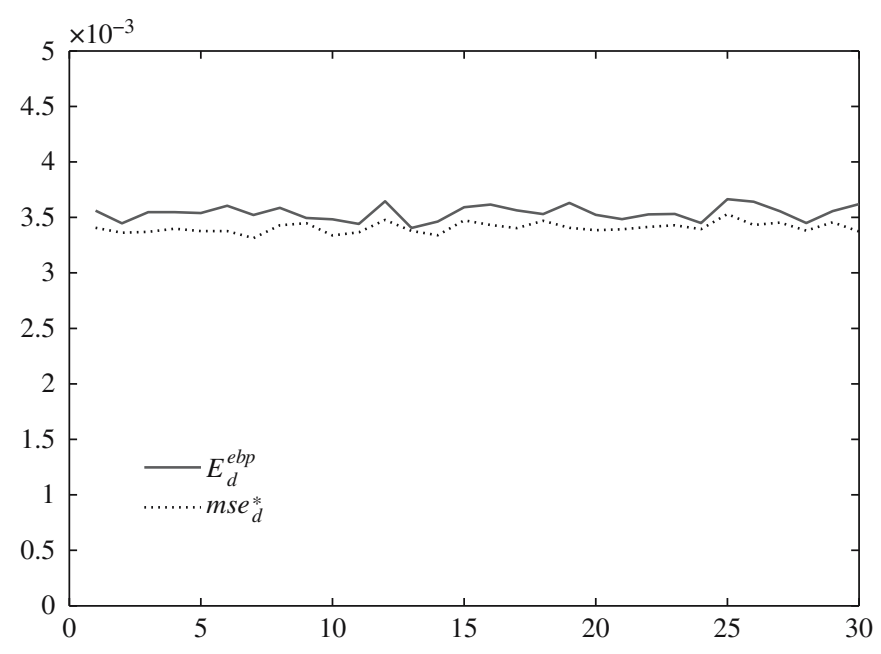

Fig. 6. Plot of $E_{d}^{e b p}$ and $m s e_{d}^{*}$ for $n_{d}=40$.

Survey to estimate poverty indicators. We use the SLCS2012 data from the Autonomous Community of Valencia (East of Spain). This region has three provinces, Alicante, Castellón and Valencia, encoded as 3, 12, and 46 by the Spanish Statistical Office. The provinces are partitioned into 9, 8, and 17 comarcas (counties) respectively, but only 8, 4, and 14 appear in the SLCS2012. The target domains are the counties, there are thus $D=34$ domains, but not all of them appear in the sample. The SLCS2012 sample size is $n=2,678$. The SCLS2012 is a two-stage area sampling design with census section as the primary units and main family addresses as the ultimate sampling units. The sampling frame is the Population Census updated from the Municipal Register.

The SLCS2012 gives information about the equivalent personal incomes, which are obtained by dividing the total household income by the equivalent total of household members. This total is calculated as a weighted sum assigning weights 1 to the first adult, 0.5 to remaining adults and 0.3 to children under 14 years of age. The weighting for obtaining the equivalent household size and income in the SLCS2012 file is done by following the instructions of EUROSTAT. The weights are based on socioeconomic theory and are not sampling weights.

The Spanish Statistical Office builds the data files of the SLCS2012 and assigns the same household equivalent income to all the household members. Because of this fact, individual-level models are not as explanatory as they hypothetically could be. One could think of fitting models at the household level. However, we cannot follow such approach because the domain-level aggregated auxiliary variables, which are used to construct a model-based census, are only available for individuals and not for households.

EUROSTAT defines the poverty line as the $60 \%$ of the median of the equivalent personal incomes in the whole country. A person is classified as poor if their equivalent personal income (denoted as $E_{d j}$ for individual $j$ of domain $d$ ) is lower than the poverty line. The poverty proportion is the proportion of people below the poverty line. The 2012 poverty line is $z=6,840$ (in euros per annum per person) for the region of Valencia. 
The poverty proportions at the domain levels are

$$
\bar{Y}_{d}=\frac{1}{N_{d}} \sum_{j=1}^{N_{d}} y_{d j}, \text { where } y_{d j}=I\left(E_{d j}<z\right), d=1, \ldots, D, \quad j=1, \ldots, N_{d} .
$$

This article estimates domain poverty proportions by using the EBPs of the corresponding weighted sums of probabilities based on unit-level logit mixed models, with random effects at domain level only. This approach requires unit-level survey data for fitting the models, and cross-classified domain level census data for constructing the EBPs. At the unit level, data is taken from the SLCS2012 and the target variable indicates whether individuals are below the poverty line (or not). As the target variable is dichotomic, we employ logit regression models.

In addition to the SLCS2012 data, we take auxiliary aggregated data from the 2012 Labour Force Survey (SLFS2012) file, which contains survey data about the labor market. The sizes of domains crossed by labor status (employed, unemployed, inactive and below 15 years old) are taken from this file. Note that by summing up in the labor categories we obtain the estimated domain sizes. We have taken the estimated domain sizes from SLFS2012 in the estimation of the EBPs. The 2012 population size for the region of Valencia, estimated from SLFS2012, is $N=4,990.277$.

We remark that we have estimated the population sizes $N_{d k}$ using SLFS2012 data and we have ignored their variability. As we have not got good covariates at the county level from Spanish administrative registers, we have instead employed SLFS2012 data and have taken the selected covariates as true aggregated values. This is a drawback of this application to real data, as it leads to underestimates of the MSE. Nevertheless, the sample size of the SLFS2012 is much higher than the one of the SLCS2012. This is why we have followed this practical approach.

For $d=1, \ldots, D, j=1, \ldots, N_{d}$, we assume that $\left.y_{d j}\right|_{v_{d}} \sim \operatorname{Bin}\left(1, p_{d j}\right)$, where $v_{1}, \ldots, v_{D}$ are i.i.d. $N(0,1)$ and

$$
\operatorname{logit}\left(p_{d j}\right)=\beta_{0}+\beta_{1} \text { employed }_{d j}+\beta_{2} \text { unemployed }_{d j}+\beta_{3} \text { inactive }_{d j}+\phi v_{d} .
$$

Table 3 presents the estimates of the model parameters and the corresponding $p$-values. We observe that the more people are employed and inactive, the smaller is the probability of being below the poverty line, and the more people are unemployed, the bigger is this probability.

Pearson residuals are calculated for each domain and covariate class. Conditionally on $v_{d}$, the sum of $y_{d j}$ over the domain $d$ and the covariate class $k$ is binomially distributed with parameters $\left(N_{d k}, p_{d k}\right)$, where $N_{d k}$ is the number of observations in the area $d$ and the covariate class $k$ and $p_{d k}$ is the corresponding model probability.

Figure 7 (left) presents a dispersion graph of residuals. The residuals are mainly located in the interval $(-2,2)$ and they do not present any visible nonrandom pattern. Figure 7 (right) presents a boxplot of the residuals. The marked residual 7 is an outlier under the standard normal distribution. We have also fitted Model (6.2) to the data without the observations belonging to the subset (domain crossed by covariate class) marked by 7 . The obtained parameter estimates do not differ significantly from the one appearing in Table 3. Therefore, we calculate the EBPs with the parameter estimates given by this table. 
Table 3. Estimates of model parameters.

\begin{tabular}{lrcr}
\hline & estimate & standard error & $p$-value \\
\hline$\beta_{0}$ & -1.1773 & 0.1473 & $1.33 \mathrm{E}-15$ \\
$\beta_{1}$ & -0.8354 & 0.1522 & $4.02 \mathrm{E}-08$ \\
$\beta_{2}$ & 0.5379 & 0.1559 & 0.000558 \\
$\beta_{3}$ & -0.4040 & 0.1445 & 0.005177 \\
\hline$\phi$ & 0.3986 & & \\
\hline
\end{tabular}

In order to check the assumption that the random effects at domain level have the standard normal distribution, we calculated the EBP $\hat{v}_{d}$ of the random effects $v_{d}$ and we present their Q-Q plot in Figure 8. We can observe quite good agreement and moreover, the Kolmogorov-Smirnov test does not reject the hypothesis $H_{0}: F_{\hat{v}_{d}}=F_{N(0,1)}$ with $p$-value equal to 0.9653 . Because random effects at finer levels are not incorporated into the model, these cannot be tested.

Table 4 presents the direct (dir) and EBP (ebp) estimates of the domain poverty proportions, the corresponding variance (var) and bootstrap MSE (mse) estimates and the relative root mean-squared errors in \% (rrmse). We have employed the simple parametric bootstrap with $B=500$ resamples. The columns labeled $n$ and $\hat{N}$ contain the SLCS2012 sample sizes and the SLFS2012-based estimated population sizes (number of individuals), respectively. The columns labeled prov and com indicate the provinces and the counties, respectively.

Table 4 shows that the EBPs have lower MSEs conditional on the model being correct than the direct estimates in areas with small sample sizes $\left(n_{d}<90\right)$ and comparable or slightly higher MSEs in the rest of domains.

Table 5 presents the EBPs (ebp) of the domain poverty proportions, the corresponding MSE estimates (mse) and the relative root mean-squared errors in \% (rrmse) for counties with zero sample size in SLCS2012. For these counties, the direct estimators are not calculable, as there is no sample.
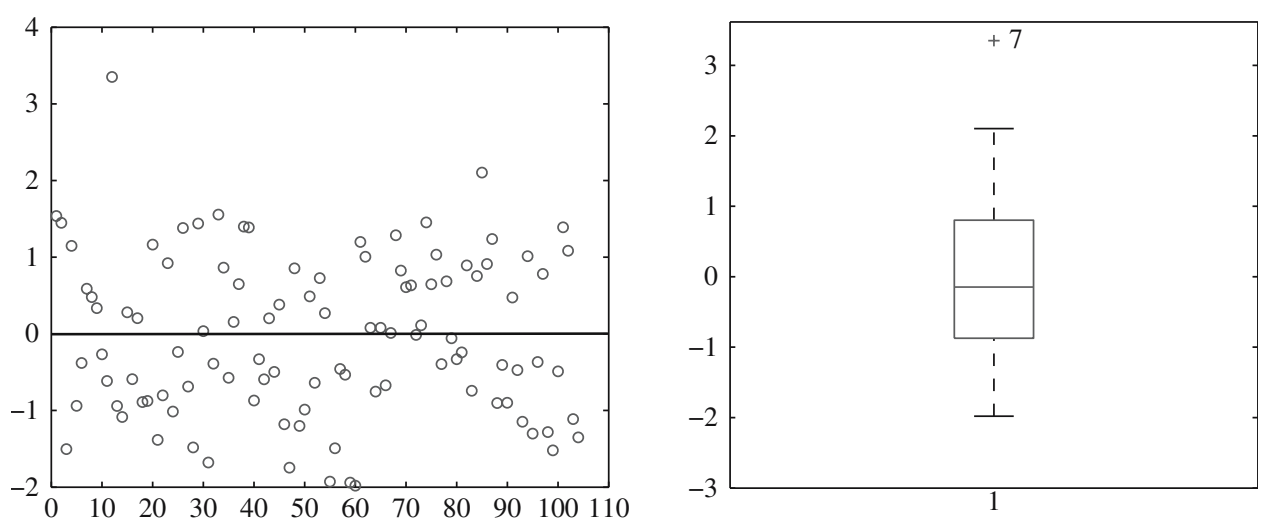

Fig. 7. Dispersion graph (left) and boxplot (right) of the residuals. 


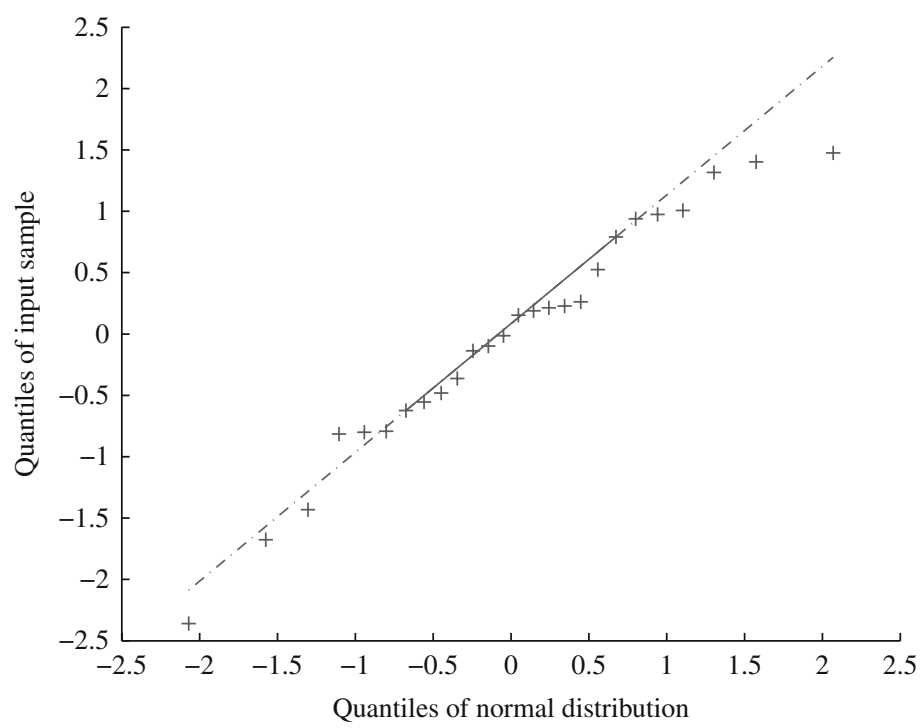

Fig. 8. $Q-Q$ plot of $\hat{v}_{d}$ with respect to $N(0,1)$ distribution.

Figure 9 (left) plots EBP and direct poverty-incidence estimates and Figure 9 (right) plots the estimated MSEs of poverty-incidence estimates. These figures show that the EBPs have a lower estimated MSE than the direct estimates.

\section{Discussion and Conclusions}

Binomial-logit regression models are a flexible class of modelling dichotomic and count variables. This work estimates poverty proportions in counties of the region of Valencia, Spain, by using a model-based unit-level logit mixed model with a domain-level random error (but without reference to survey clustering or survey weighting), and using predictors of weighted sums of probabilities. We fit the model by the method of simulated moments. We consider the EBP and two plug-in estimators and we compare them in a simulation study, based (due to computational complexity) on a small sample and a very small census.

The assumed binomial-logit model with normally distributed domain random effects is widely used in small-area estimation when the parameter of interest is a proportion or a sum of probabilities. The model can be extended from $v_{d} \sim$ Normal to $v_{d} \sim F$, where $F$ is a cumulative distribution function with support on the real line. The extension to heteroscedasticity can also be formulated. If we do this however, we would have to study which of the properties of the binomial logit-normal models still hold in the new model, or how they are modified. Working with a well-studied model has the advantage of having mathematical (e.g., asymptotic) results on the model parameter estimators. In particular, some properties established by Jiang (1998, 2003) and Jiang and Lahiri (2001) are employed in the article to derive the approximation to the model-based MSE of the EBP, conditional on a specified model and without random effects at levels finer than domain.

For the EBP, we derive a model-based MSE and introduce four estimators. The first two are analytic estimators without and with bias correction of the second order. The third and 
Table 4. Direct and EBP poverty-proportions estimates.

\begin{tabular}{lrrr|rrr|crr}
\hline prov & rom & $n$ & $\hat{N}$ & dir & var & rrmse & ebp & mse & rrmse \\
\hline 46 & 17 & 12 & 35415 & 0.3085 & 0.0164 & 41.52 & 0.1957 & 0.0035 & 30.23 \\
12 & 7 & 17 & 28715 & 0.3010 & 0.0113 & 35.32 & 0.2231 & 0.0031 & 25.11 \\
46 & 12 & 18 & 88625 & 0.0000 & 0.0076 & & 0.1384 & 0.0032 & 40.94 \\
46 & 22 & 18 & 30672 & 0.5010 & 0.0101 & 20.04 & 0.2405 & 0.0030 & 22.76 \\
3 & 31 & 37 & 151846 & 0.0133 & 0.0051 & 536.71 & 0.1149 & 0.0029 & 46.60 \\
46 & 18 & 37 & 55440 & 0.1103 & 0.0049 & 63.65 & 0.1582 & 0.0027 & 32.76 \\
46 & 23 & 45 & 75157 & 0.2490 & 0.0051 & 28.65 & 0.1982 & 0.0026 & 25.87 \\
46 & 21 & 47 & 53869 & 0.2454 & 0.0036 & 24.53 & 0.2528 & 0.0028 & 21.06 \\
12 & 3 & 56 & 86580 & 0.2993 & 0.0034 & 19.56 & 0.2796 & 0.0026 & 18.34 \\
3 & 34 & 66 & 282076 & 0.1216 & 0.0032 & 46.40 & 0.1659 & 0.0025 & 29.90 \\
46 & 24 & 66 & 86138 & 0.1251 & 0.0025 & 39.82 & 0.1615 & 0.0025 & 31.14 \\
46 & 25 & 75 & 177618 & 0.1711 & 0.0031 & 32.49 & 0.2009 & 0.0021 & 23.04 \\
3 & 28 & 82 & 76849 & 0.3962 & 0.0027 & 13.05 & 0.1817 & 0.0021 & 25.31 \\
3 & 29 & 85 & 228083 & 0.0868 & 0.0025 & 57.58 & 0.1270 & 0.0020 & 35.56 \\
12 & 6 & 88 & 182172 & 0.1823 & 0.0020 & 24.69 & 0.1855 & 0.0024 & 26.42 \\
3 & 27 & 92 & 125445 & 0.2995 & 0.0019 & 14.39 & 0.2688 & 0.0022 & 17.33 \\
46 & 16 & 109 & 139697 & 0.2995 & 0.0022 & 15.58 & 0.2617 & 0.0021 & 17.44 \\
46 & 11 & 109 & 179960 & 0.1984 & 0.0016 & 20.37 & 0.1543 & 0.0022 & 30.71 \\
12 & 5 & 117 & 251429 & 0.3141 & 0.0029 & 17.19 & 0.1812 & 0.0021 & 25.05 \\
46 & 20 & 124 & 260449 & 0.0223 & 0.0012 & 158.11 & 0.0747 & 0.0019 & 58.38 \\
46 & 13 & 125 & 184704 & 0.1265 & 0.0014 & 29.11 & 0.1456 & 0.0019 & 30.25 \\
3 & 30 & 147 & 239247 & 0.1774 & 0.0016 & 22.31 & 0.2021 & 0.0016 & 19.60 \\
3 & 33 & 154 & 266020 & 0.2967 & 0.0011 & 11.20 & 0.2898 & 0.0017 & 14.25 \\
46 & 14 & 213 & 373164 & 0.2527 & 0.0010 & 12.52 & 0.2509 & 0.0012 & 13.76 \\
3 & 32 & 298 & 467602 & 0.1830 & 0.0006 & 13.24 & 0.1568 & 0.0011 & 21.00 \\
46 & 15 & 441 & 781222 & 0.2134 & 0.0005 & 10.08 & 0.1926 & 0.0013 & 18.41 \\
\hline & & & & & & & & &
\end{tabular}

fourth estimators are based on a parametric bootstrap. We analyze the behavior of the proposed estimators in a small simulation study. Estimating the bias correction term is computationally intensive and the results of the analytic estimators without and with bias correction are quite similar for very small domain sample sizes, although this conclusion may require modification if cluster and household level random effects are included or survey weighting used. The MSE analytical estimators are consistent, but they are not practical for medium- and large-domain sample sizes, because the calculation of the term

Table 5. EBP poverty-proportions estimates for counties without sample $\left(n_{d}=0\right)$.

\begin{tabular}{lrr|ccc}
\hline prov & com & $\hat{N}$ & ebp & mse & rrmse \\
\hline 3 & 26 & 22310 & 0.1943 & 0.0034 & 30.13 \\
12 & 1 & 7819 & 0.1680 & 0.0024 & 29.36 \\
12 & 2 & 6868 & 0.1712 & 0.0029 & 31.70 \\
12 & 4 & 16109 & 0.1780 & 0.0022 & 26.23 \\
12 & 8 & 5529 & 0.1781 & 0.0035 & 33.11 \\
46 & 9 & 1826 & 0.1879 & 0.0029 & 28.52 \\
46 & 10 & 14400 & 0.1964 & 0.0036 & 30.69 \\
46 & 19 & 7222 & 0.1920 & 0.0029 & 28.28 \\
\hline
\end{tabular}



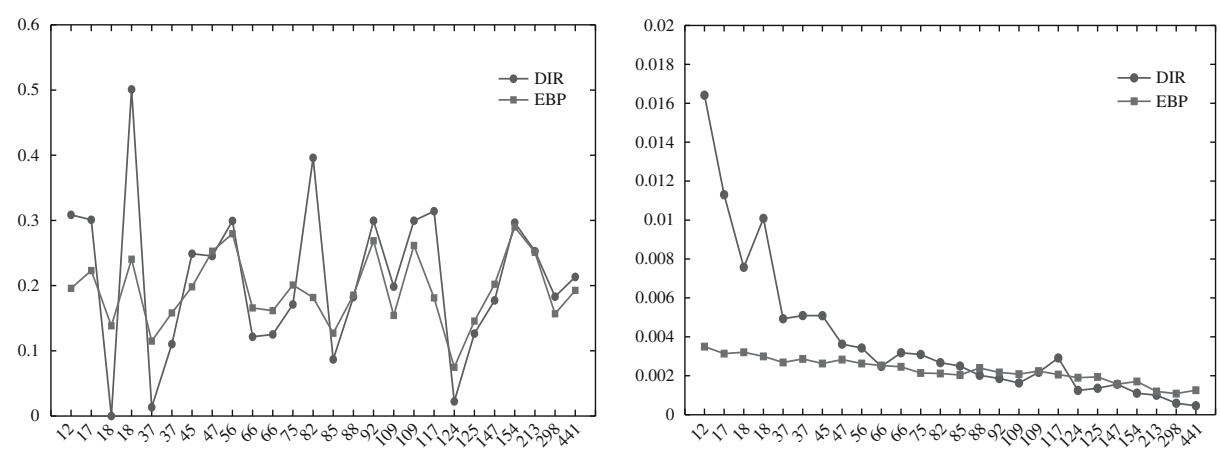

Fig. 9. EBP and direct poverty-proportions estimates (left) and estimated MSEs (right).

$g_{d}$ involves iteratively finding the $\left(\begin{array}{l}n_{d} \\ j\end{array}\right)$ subsets of size $j$ of a set of size $n_{d}$. This problem requires a high-speed computer with large RAM memory for $n_{d} \geq 40$, so would be difficult to implement for most real-world applications where samples in each surveyed domain are almost always much larger.

Our simulations are computationally intensive in two senses. First, they are carried out at the unit level as we deal with unit-level models and not at the area level. Second and more importantly, all the considered methodology is computationally expensive. The fitting method (MSM) proposed by Jiang (1998) has nice asymptotic properties and allows approximating the MSE of the EBP. However, it requires solving a nonlinear system of equations by using a Newton-Raphson algorithm, where the components of the updating formula have to be approximated by Monte Carlo simulation in each algorithm step. Therefore, it is not a high-speed procedure. The calculation of the EBPs for $d=1, \ldots, D$, requires evaluating integrals by Monte Carlo simulation. This is again time consuming. The calculation of the MSE estimators (analytic and bootstrap) are even more time consuming than the calculation of the EBPs.

The computational burden is not a major problem for the application of the model to real data (with only one sample) provided domain sizes are kept small, but it is a major problem for the 1,000 samples in the simulation (which is why sample and population sizes have been kept very small there). For example, we parallelized Simulation 3 in several computers and they ran for two weeks before obtaining the results. This is why we have simplified the simulation scenario and have implemented a reduced-in-size version. The small sample and population sizes necessary for the simulation do however reduce general applicability of the results.

Jiang and Lahiri (2001) did not present any application to data. This article shows that the EBP methodology may with further development be applicable to real small-area estimation problems. One exception is Jiang and Lahiri's MSE estimator, which would instead need an alternative computationally feasible approach. As a good alternative, we suggest the introduced parametric bootstrap procedure, which does not have the same computational drawbacks, is easy to implement, and has generally good behavior.

In the application to poverty data from the SLCS2012, we use the EBPs to estimate poverty proportions. We take the model-based MSE for a synthetic estimator estimated via the parametric bootstrap as a performance measure. The units are taken to be individuals, 
not households, and the model is fitted without adjustment for the complex structure of the survey design. From the point of view of modelling the survey data, it would be better to work with households as sampling units (instead of individuals), because some survey variables (like income) are household variables. The problem appears when calculating the EBPs. In the Spanish case, we do not have a full unit-record census file and we instead need to rely on categorical covariates only, taken from census cross tabulations. Consequently, the population sizes available in covariates classes crossed by domains are individual based. The corresponding information is not available for households. This is the reason we have treated individuals as sampling units.

The introduced model has only one random effect for domains. In future, we might consider hierarchical models with random effects on the different levels of the hierarchy. This would allow more accurate modelling, including household effects. The model proposed in this article is however purely model-based, without reference to the structure of the complex survey design, so does not include stratification, clustering or survey-based weighting. Therefore, model assumptions need to be comprehensively checked to the extent possible by using diagnostic tools, like graphical methods, testing procedures and residual analysis. For a simple random sample, at unit level the $\left\{y_{d j}\right\}$ are independent, conditional on the model and the domain-level random effects. The same is true if we consider stratified random sampling with domains nested within strata. For more complex designs (like a design clustered within domains) this assumption will not hold.

Another issue that remains to be studied is how survey weighting could be included in the analysis. For example, how best to introduce the survey weights in the model-based methodology to reduce the design bias of predictors? The difficult problems linked with survey-design issues for small-area predictors based on binomial-logit mixed models will require further research.

\section{Appendix}

\section{A.1. Components of MM Newton-Raphson Algorithm}

The MM Newton-Raphson algorithm is specified if we calculate the expectations appearing in $\boldsymbol{f}(\boldsymbol{\theta})$ and its partial derivatives. The expectation of $y_{d j}$ is

$$
E_{\boldsymbol{\theta}}\left[y_{d j}\right]=E_{v}\left[E_{\boldsymbol{\theta}}\left[y_{d j} \mid \boldsymbol{v}\right]\right]=E_{v}\left[m_{d j} p_{d j}\right]
$$

and the corresponding derivatives are

$$
\frac{\partial E_{\boldsymbol{\theta}}\left[y_{d j}\right]}{\partial \beta_{k}}=E_{v}\left[m_{d j} p_{d j}\left(1-p_{d j}\right) x_{d j k}\right], \quad \frac{\partial E_{\boldsymbol{\theta}}\left[y_{d j}\right]}{\partial \phi}=E_{v}\left[m_{d j} p_{d j}\left(1-p_{d j}\right) v_{d}\right] .
$$

The expectation of $y_{d .}^{2}$ is $E_{\boldsymbol{\theta}}\left[y_{d .}^{2}\right]=E_{v}\left[E_{\boldsymbol{\theta}}\left[y_{d}^{2} \mid \boldsymbol{v}\right]\right]$, where

$$
\begin{gathered}
y_{d .}^{2}=\sum_{j=1}^{n_{d}} y_{d j}^{2}+\sum_{j_{1} \neq j_{2}}^{n_{d}} y_{d j_{1}} y_{d j_{2}}, \\
E_{\boldsymbol{\theta}}\left[y_{d j}^{2} \mid \boldsymbol{v}\right]=\operatorname{var}_{\boldsymbol{\theta}}\left[y_{d j} \mid \boldsymbol{v}\right]+E_{\boldsymbol{\theta}}^{2}\left[y_{d j} \mid \boldsymbol{v}\right]=m_{d j} p_{d j}\left(1-p_{d j}\right)+m_{d j}^{2} p_{d j}^{2} .
\end{gathered}
$$


Therefore, we have

$$
E_{\boldsymbol{\theta}}\left[y_{d .}^{2}\right]=E_{v}\left[\sum_{j=1}^{n_{d}} m_{d j} p_{d j}\left(1-p_{d j}\right)+\left(\sum_{j=1}^{n_{d}} m_{d j} p_{d j}\right)^{2}\right] .
$$

Let us define $\xi_{d}=\sum_{j=1}^{n_{d}} m_{d j} p_{d j}$. The derivatives of $E_{\boldsymbol{\theta}}\left[y_{d}^{2}\right]$ are

$$
\begin{aligned}
& \frac{\partial E_{\boldsymbol{\theta}}\left[y_{d .}^{2}\right]}{\partial \beta_{k}}=\sum_{j=1}^{n_{d}} E_{v}\left[m_{d j} p_{d j}\left(1-p_{d j}\right)\left\{1-2\left(p_{d j}-\xi_{d}\right)\right\} x_{d j k}\right], \\
& \frac{\partial E_{\boldsymbol{\theta}}\left[y_{d .}^{2}\right]}{\partial \phi}=\sum_{j=1}^{n_{d}} E_{v}\left[m_{d j} p_{d j}\left(1-p_{d j}\right)\left\{1-2\left(p_{d j}-\xi_{d}\right)\right\} v_{d}\right] .
\end{aligned}
$$

The elements of the matrix of first partial derivatives are

$$
\begin{aligned}
H_{k \ell} & =\frac{\partial f_{k}(\boldsymbol{\theta})}{\partial \theta_{\ell}}=\sum_{d=1}^{D} \sum_{j=1}^{n_{d}} \frac{\partial E_{\boldsymbol{\theta}}\left[y_{d j}\right]}{\partial \theta_{\ell}} x_{d j k}, \quad k=1, \ldots, p, \ell=1, \ldots, p+1, \\
H_{p+1 \ell} & =\frac{\partial f_{p+1}(\boldsymbol{\theta})}{\partial \theta_{\ell}}=\sum_{d=1}^{D} \frac{\partial E_{\boldsymbol{\theta}}\left[y_{d .}^{2}\right]}{\partial \theta_{\ell}}, \quad \ell=1, \ldots, p+1,
\end{aligned}
$$

where $\theta_{1}=\beta_{1}, \ldots, \theta_{p}=\beta_{p}, \theta_{p+1}=\phi$. The expectations appearing in $\boldsymbol{f}(\boldsymbol{\theta})$ and $\boldsymbol{H}(\boldsymbol{\theta})$ can be approximated by Monte Carlo simulation.

\section{A.2. Proof of Proposition 4.1}

A first-order multivariate Taylor expansion of $\boldsymbol{M}(\hat{\boldsymbol{\theta}})$ around $\boldsymbol{\theta}$ yields

$$
\boldsymbol{M}(\hat{\boldsymbol{\theta}})=\boldsymbol{M}(\boldsymbol{\theta})+\left(\frac{\partial}{\partial \boldsymbol{\theta}} \boldsymbol{M}(\boldsymbol{\theta})\right)(\hat{\boldsymbol{\theta}}-\boldsymbol{\theta})+o(\|\hat{\boldsymbol{\theta}}-\boldsymbol{\theta}\|) .
$$

Therefore

$$
\hat{\boldsymbol{\theta}}-\boldsymbol{\theta}=\left(\frac{\partial}{\partial \boldsymbol{\theta}} \boldsymbol{M}(\boldsymbol{\theta})\right)^{-1}(\boldsymbol{M}(\hat{\boldsymbol{\theta}})-\boldsymbol{M}(\boldsymbol{\theta}))+o(\|\hat{\boldsymbol{\theta}}-\boldsymbol{\theta}\|) .
$$

Let us consider a univariate second order Taylor expansion of $\boldsymbol{M}_{k}(\hat{\boldsymbol{\theta}})$ around $\boldsymbol{\theta}$ and substitute (A.1) in the quadratic term, that is

$$
\begin{aligned}
\boldsymbol{M}_{k}(\hat{\boldsymbol{\theta}})= & \boldsymbol{M}_{k}(\boldsymbol{\theta})+\left(\frac{\partial}{\partial \boldsymbol{\theta}} \boldsymbol{M}_{k}(\boldsymbol{\theta})\right)^{\prime}(\hat{\boldsymbol{\theta}}-\boldsymbol{\theta})+\frac{1}{2}(\hat{\boldsymbol{\theta}}-\boldsymbol{\theta})^{\prime}\left(\frac{\partial^{2}}{\partial \boldsymbol{\theta}^{2}} M_{k}(\boldsymbol{\theta})\right) \\
& (\hat{\boldsymbol{\theta}}-\boldsymbol{\theta})+o\left(\|\hat{\boldsymbol{\theta}}-\boldsymbol{\theta}\|^{2}\right) \\
= & \boldsymbol{M}_{k}(\boldsymbol{\theta})+\left(\frac{\partial}{\partial \boldsymbol{\theta}} \boldsymbol{M}_{k}(\boldsymbol{\theta})\right)^{\prime}(\hat{\boldsymbol{\theta}}-\boldsymbol{\theta})+\frac{1}{2}(\boldsymbol{M}(\hat{\boldsymbol{\theta}})-\boldsymbol{M}(\boldsymbol{\theta}))^{\prime}\left(\left(\frac{\partial}{\partial \boldsymbol{\theta}} \boldsymbol{M}(\boldsymbol{\theta})\right)^{-1}\right)^{\prime} \\
& \cdot\left(\frac{\partial^{2}}{\partial \boldsymbol{\theta}^{2}} M_{k}(\boldsymbol{\theta})\right)\left(\frac{\partial}{\partial \boldsymbol{\theta}} \boldsymbol{M}(\boldsymbol{\theta})\right)^{-1}(\boldsymbol{M}(\hat{\boldsymbol{\theta}})-\boldsymbol{M}(\boldsymbol{\theta}))+o\left(\|\hat{\boldsymbol{\theta}}-\boldsymbol{\theta}\|^{2}\right) .
\end{aligned}
$$


The corresponding multivariate Taylor expansion of $\boldsymbol{M}(\hat{\boldsymbol{\theta}})$ around $\boldsymbol{\theta}$ is

$$
\begin{aligned}
\boldsymbol{M}(\hat{\boldsymbol{\theta}})= & \boldsymbol{M}(\boldsymbol{\theta})+\left(\frac{\partial}{\partial \boldsymbol{\theta}} \boldsymbol{M}(\boldsymbol{\theta})\right)(\hat{\boldsymbol{\theta}}-\boldsymbol{\theta})+\frac{1}{2} \underset{1 \leq k \leq p+1}{\operatorname{col}}\left((\boldsymbol{M}(\hat{\boldsymbol{\theta}})-\boldsymbol{M}(\boldsymbol{\theta}))^{\prime}\left(\left(\frac{\partial}{\partial \boldsymbol{\theta}} \boldsymbol{M}(\boldsymbol{\theta})\right)^{-1}\right)^{\prime}\right. \\
& \left.\cdot\left(\frac{\partial^{2}}{\partial \boldsymbol{\theta}^{2}} M_{k}(\boldsymbol{\theta})\right)\left(\frac{\partial}{\partial \boldsymbol{\theta}} \boldsymbol{M}(\boldsymbol{\theta})\right)^{-1}(\boldsymbol{M}(\hat{\boldsymbol{\theta}})-\boldsymbol{M}(\boldsymbol{\theta}))\right)+o\left(\|\hat{\boldsymbol{\theta}}-\boldsymbol{\theta}\|^{2}\right) \\
= & \boldsymbol{M}(\boldsymbol{\theta})+\left(\frac{\partial}{\partial \boldsymbol{\theta}} \boldsymbol{M}(\boldsymbol{\theta})\right)(\hat{\boldsymbol{\theta}}-\boldsymbol{\theta})+\frac{1}{2 D} \boldsymbol{q}_{D}+o\left(\|\hat{\boldsymbol{\theta}}-\boldsymbol{\theta}\|^{2}\right) .
\end{aligned}
$$

Therefore

$$
\hat{\boldsymbol{\theta}}-\boldsymbol{\theta}=\left(\frac{\partial}{\partial \boldsymbol{\theta}} \boldsymbol{M}(\boldsymbol{\theta})\right)^{-1}\left[(\boldsymbol{M}(\hat{\boldsymbol{\theta}})-\boldsymbol{M}(\boldsymbol{\theta}))-\frac{1}{2 D} \boldsymbol{q}_{D}\right]+o\left(\|\hat{\boldsymbol{\theta}}-\boldsymbol{\theta}\|^{2}\right) .
$$

By substituting (A.2) in (4.3), we obtain

$$
\begin{aligned}
b_{d}(\boldsymbol{\theta})= & \left(\frac{\partial}{\partial \boldsymbol{\theta}} g_{d}(\boldsymbol{\theta})\right)^{\prime} D\left(\frac{\partial}{\partial \boldsymbol{\theta}} \boldsymbol{M}(\boldsymbol{\theta})\right)^{-1}\left\{E[\boldsymbol{M}(\hat{\boldsymbol{\theta}})-\boldsymbol{M}(\boldsymbol{\theta})]-\frac{1}{2 D} E\left[\boldsymbol{q}_{D}\right]\right\} \\
& +\frac{1}{2} E\left[\sqrt{D}\left[(\boldsymbol{M}(\hat{\boldsymbol{\theta}})-\boldsymbol{M}(\boldsymbol{\theta}))^{\prime}-\frac{1}{2 D} \boldsymbol{q}^{\prime}{ }_{D}\right]\left(\left(\frac{\partial}{\partial \boldsymbol{\theta}} \boldsymbol{M}(\boldsymbol{\theta})\right)^{-1}\right)^{\prime}\left(\frac{\partial^{2}}{\partial \boldsymbol{\theta}^{2}} g_{d}(\boldsymbol{\theta})\right)\right. \\
& \left.\cdot\left(\frac{\partial}{\partial \boldsymbol{\theta}} \boldsymbol{M}(\boldsymbol{\theta})\right)^{-1} \sqrt{D}\left[(\boldsymbol{M}(\hat{\boldsymbol{\theta}})-\boldsymbol{M}(\boldsymbol{\theta}))-\frac{1}{2 D} \boldsymbol{q}_{D}\right]\right]+D o\left(\|\hat{\boldsymbol{\theta}}-\boldsymbol{\theta}\|^{2}\right) .
\end{aligned}
$$

On the one hand, we substitute $\boldsymbol{M}(\hat{\boldsymbol{\theta}})$ by $\hat{\boldsymbol{M}}$, so that $E[\boldsymbol{M}(\hat{\boldsymbol{\theta}})-\boldsymbol{M}(\boldsymbol{\theta})]=E[\hat{\boldsymbol{M}}-\boldsymbol{M}(\boldsymbol{\theta})]=0$. All the quadratic forms in the second summand containing $\boldsymbol{q}_{D}$ are $o(1)$. Therefore

$$
\begin{aligned}
b_{d}(\boldsymbol{\theta})= & -\frac{1}{2}\left(\frac{\partial}{\partial \boldsymbol{\theta}} g_{d}(\boldsymbol{\theta})\right)^{\prime}\left(\frac{\partial}{\partial \boldsymbol{\theta}} \boldsymbol{M}(\boldsymbol{\theta})\right)^{-1} E\left[\boldsymbol{q}_{D}\right] \\
& +\frac{1}{2} E\left[\sqrt{D}(\boldsymbol{M}(\hat{\boldsymbol{\theta}})-\boldsymbol{M}(\boldsymbol{\theta}))^{\prime}\left(\left(\frac{\partial}{\partial \boldsymbol{\theta}} \boldsymbol{M}(\boldsymbol{\theta})\right)^{-1}\right)^{\prime}\left(\frac{\partial^{2}}{\partial \boldsymbol{\theta}^{2}} g_{d}(\boldsymbol{\theta})\right)\right. \\
& \left.\cdot\left(\frac{\partial}{\partial \boldsymbol{\theta}} \boldsymbol{M}(\boldsymbol{\theta})\right)^{-1} \sqrt{D}(\boldsymbol{M}(\hat{\boldsymbol{\theta}})-\boldsymbol{M}(\boldsymbol{\theta}))\right]+o(1) \\
= & \frac{1}{2}\left\{E\left[r_{D, d}\right]-\left(\frac{\partial}{\partial \boldsymbol{\theta}} g_{d}(\boldsymbol{\theta})\right)^{\prime}\left(\frac{\partial}{\partial \boldsymbol{\theta}} \boldsymbol{M}(\boldsymbol{\theta})\right)^{-1} E\left[\boldsymbol{q}_{D}\right]\right\}+o(1)=B_{d}(\boldsymbol{\theta})+o(1) .
\end{aligned}
$$

\section{A.3. MSE Components}

The MSE estimators $m s e^{0}\left(\hat{\mu}_{d}\right)$ and $m s e^{1}\left(\hat{\mu}_{d}\right)$ given by (4.4) have the components $g_{d}, c_{d}$ and $B_{d}$. This appendix gives algorithms for approximating them. 
The term $g_{d}(\hat{\boldsymbol{\theta}})$ can be approximated by $\hat{g}_{d}(\hat{\boldsymbol{\theta}})=\hat{E}\left[\hat{\mu}_{d}^{2}(\hat{\boldsymbol{\theta}})\right]-\hat{E}\left[\hat{\mu}_{d}^{2}(\hat{\boldsymbol{\theta}})\right]$, where the expectations are calculated by Monte Carlo simulation. This is to say, generate $v_{d}^{(s)}$ to be i.i.d. $N(0,1)$ random variables and $v_{d}^{(S+s)}=-v_{d}^{(s)}, s=1, \ldots, S$, and calculate

$$
\begin{aligned}
& \hat{E}\left[\mu_{d}^{2}(\hat{\boldsymbol{\theta}})\right]=\frac{1}{2 S} \sum_{s=1}^{2 S}\left(\sum_{k=1}^{K} N_{d k} \frac{\exp \left\{\boldsymbol{z}_{k} \hat{\boldsymbol{\beta}}+\hat{\boldsymbol{\phi}} v_{d}^{(s)}\right\}}{1+\exp \left\{\boldsymbol{z}_{k} \hat{\boldsymbol{\beta}}+\hat{\boldsymbol{\phi}} v_{d}^{(s)}\right\}}\right)^{2}, \\
& \hat{E}\left[\hat{\mu}_{d}^{2}(\hat{\boldsymbol{\theta}})\right]=\sum_{j=0}^{n_{d}} \hat{\psi}_{d}^{2}(j, \hat{\boldsymbol{\theta}}) \hat{p}_{d}(j, \hat{\boldsymbol{\theta}}),
\end{aligned}
$$

where $\hat{\psi}_{d}\left(y_{d}, \hat{\boldsymbol{\theta}}\right)=\sum_{k=1}^{K} N_{d k} \frac{\hat{A}_{d k}^{z}}{\hat{C}_{d}}$ and

$\hat{p}_{d}(j, \hat{\boldsymbol{\theta}})=\sum_{\boldsymbol{y} \in S_{n_{d}, j}}\left\{\exp \left\{\sum_{i=1}^{n_{d}} y_{i} \boldsymbol{x}_{d i} \hat{\boldsymbol{\beta}}\right\} \frac{1}{2 S} \sum_{s=1}^{2 S} \exp \left\{j \hat{\boldsymbol{\phi}} v_{d}^{(s)}-\sum_{i=1}^{n_{d}} \log \left[1+\exp \left\{\boldsymbol{x}_{d i} \hat{\boldsymbol{\beta}}+\hat{\boldsymbol{\phi}} v_{d}^{(s)}\right\}\right]\right\}\right\}$.

The term $c_{d}(\hat{\boldsymbol{\theta}})$ can be approximated by

$$
\hat{c}_{d}(\hat{\boldsymbol{\theta}})=\sum_{j=1}^{n_{d}}\left(\frac{\partial}{\partial \boldsymbol{\theta}} \hat{\psi}_{d}(j, \hat{\boldsymbol{\theta}})\right)^{\prime} \hat{\boldsymbol{V}}(\hat{\boldsymbol{\theta}})\left(\frac{\partial}{\partial \boldsymbol{\theta}} \hat{\psi}_{d}(j, \hat{\boldsymbol{\theta}})\right) \hat{p}_{d}(j, \hat{\boldsymbol{\theta}})
$$

where $\hat{\boldsymbol{V}}(\hat{\boldsymbol{\theta}})=D \widehat{\operatorname{var}}_{B}(\hat{\boldsymbol{\theta}})$.

The bias correction term $B_{d}(\boldsymbol{\theta})$ can be approximated by parametric bootstrap, that is

1. Fit the model to the sample and calculate $\hat{\boldsymbol{\theta}}, \boldsymbol{R}_{d}(\hat{\boldsymbol{\theta}}), \boldsymbol{M}(\hat{\boldsymbol{\theta}})$ and $\boldsymbol{Q}_{k}(\hat{\boldsymbol{\theta}})$.

2. Generate bootstrap samples $\left\{y_{d j}^{(b)}: d=1, \ldots, D, j=1, \ldots, n_{d}\right\}, b=1, \ldots, B$, from the fitted model.

3. For each bootstrap sample, calculate $\Delta_{D}^{(b)}=\sqrt{D}\left(\hat{\boldsymbol{M}}^{(b)}-\boldsymbol{M}(\hat{\boldsymbol{\theta}})\right)$, where $\hat{\boldsymbol{M}}^{(b)}$ $=\operatorname{col}_{1 \leq k \leq p+1}\left(\hat{M}_{k}^{(b)}\right), \hat{M}_{k}^{(b)}=\sum_{d=1}^{D} \sum_{j=1}^{n_{d}} y_{d j}^{(b)} x_{d j k}, k=1, \ldots, p, \hat{M}_{p+1}^{(b)}=\sum_{d=1}^{D}\left(y_{d .}^{(b)}\right)^{2}$, and calculate

$$
r_{D, d}^{(b)}=\Delta_{D}^{(b)^{\prime}} \boldsymbol{R}_{d}(\hat{\boldsymbol{\theta}}) \Delta_{D}^{(b)}, \quad q_{D k}^{(b)}=\Delta_{D}^{(b)^{\prime}} \boldsymbol{Q}_{k}(\hat{\boldsymbol{\theta}}) \Delta_{D}^{(b)}, \quad \boldsymbol{q}_{D}^{(b)}=\underset{1 \leq k \leq p+1}{\operatorname{col}}\left(q_{D k}^{(b)}\right)
$$

4. Calculate $\hat{E}_{B}\left[r_{D, d}\right]=\frac{1}{B} \sum_{b=1}^{B} r_{D, d}^{(b)}, \hat{E}_{B}\left[\boldsymbol{q}_{D}\right]=\frac{1}{B} \sum_{b=1}^{B} \boldsymbol{q}_{D}^{(b)}$.

5. Output: $\hat{B}_{d}(\hat{\boldsymbol{\theta}})=\frac{1}{2}\left\{\hat{E}_{B}\left[r_{D, d}\right]-\left(\frac{\partial}{\partial \boldsymbol{\theta}} \hat{g}_{d}(\hat{\boldsymbol{\theta}})\right)^{\prime}\left(\frac{\partial}{\partial \boldsymbol{\theta}} \boldsymbol{M}(\hat{\boldsymbol{\theta}})\right)^{-1} \hat{E}_{B}\left[\boldsymbol{q}_{D}\right]\right\}$.

\section{A.4. Derivatives Needed to Calculate MSE Components}

To calculate the approximation of the terms $c_{d}$ and particularly $B_{d}$ we need to evaluate first and second order derivatives of $g_{d}(\boldsymbol{\theta})$ and $M_{k}(\boldsymbol{\theta})$ (cf. Proposition 4.1.). In this appendix we present formulas for derivatives of these and other necessary terms with respect to parameters $\beta_{r}, r=1, \ldots, p$, in the case of first order and with respect to $\beta_{s} \beta_{r}$, $r, s=1, \ldots, p$, in the case of second order. The derivatives with respect to $\phi, \beta_{r} \phi$ and $\phi^{2}$ can be obtained in a similar form and they are omitted here. 


\section{A.4.1. Derivatives of $\boldsymbol{M}(\boldsymbol{\theta})$}

We recall that $\boldsymbol{M}(\boldsymbol{\theta}) \underset{1 \leq k \leq p+1}{=\operatorname{col}}\left(M_{k}(\boldsymbol{\theta})\right)$, where

$$
\begin{aligned}
M_{k}(\boldsymbol{\theta}) & =\sum_{d=1}^{D} \sum_{j=1}^{n_{d}} E_{\boldsymbol{\theta}}\left[y_{d j}\right] x_{d j k}=\sum_{d=1}^{D} \sum_{j=1}^{n_{d}} x_{d j k} E_{v}\left[p_{d j}\right], \\
M_{p+1}(\boldsymbol{\theta}) & =\sum_{d=1}^{D} E_{\boldsymbol{\theta}}\left[y_{d .}^{2}\right]=\sum_{d=1}^{D} E_{v}\left[\sum_{j=1}^{n_{d}} p_{d j}\left(1-p_{d j}\right)+\left(\sum_{j=1}^{n_{d}} p_{d j}\right)^{2}\right] .
\end{aligned}
$$

Let us define $\xi_{d}=\sum_{j=1}^{n_{d}} p_{d j}$ for $p_{d j}$ given in (2.3) and note that

$$
\frac{\partial p_{d j}}{\partial \beta_{r}}=x_{d j r} p_{d j}\left(1-p_{d j}\right), \quad \frac{\partial p_{d j}\left(1-p_{d j}\right)}{\partial \beta_{r}}=x_{d j r} p_{d j}\left(1-p_{d j}\right)\left(1-2 p_{d j}\right)
$$

and

$$
\frac{\partial\left(p_{d j}-\xi_{d}\right)}{\partial \beta_{s}}=x_{d j s} p_{d j}\left(1-p_{d j}\right)-\sum_{i=1}^{n_{d}} p_{d i}\left(1-p_{d i}\right) x_{d i s}
$$

The first and second order partial derivatives of $M_{k}(\boldsymbol{\theta})$ are for $k, r, s=1, \ldots, p$

$$
\begin{gathered}
\frac{\partial M_{k}}{\partial \beta_{r}}=\sum_{d=1}^{D} \sum_{j=1}^{n_{d}} x_{d j k} x_{d j r} E_{v}\left[p_{d j}\left(1-p_{d j}\right)\right], \\
\frac{\partial M_{p+1}}{\partial \beta_{r}}=\sum_{d=1}^{D} \sum_{j=1}^{n_{d}} x_{d j r} E_{v}\left[p_{d j}\left(1-p_{d j}\right)\left\{1-2\left(p_{d j}-\xi_{d}\right)\right\}\right], \\
\frac{\partial^{2} M_{k}}{\partial \beta_{s} \partial \beta_{r}}=\sum_{d=1}^{D} \sum_{j=1}^{n_{d}} x_{d j k} x_{d j r} x_{d j s} E_{v}\left[p_{d j}\left(1-p_{d j}\right)\left(1-2 p_{d j}\right)\right], \\
\frac{\partial^{2} M_{p+1}}{\partial \beta_{s} \partial \beta_{r}}=\sum_{d=1}^{D} \sum_{j=1}^{n_{d}} x_{d j r} E_{v}\left[x_{d j s} p_{d j}\left(1-p_{d j}\right)\left(1-2 p_{d j}\right)\left\{1-2\left(p_{d j}-\xi_{d}\right)\right\}\right. \\
\left.-2 p_{d j}^{2}\left(1-p_{d j}\right)^{2} x_{d j s}+2 p_{d j}\left(1-p_{d j}\right) \sum_{i=1}^{n_{d}} p_{d i}\left(1-p_{d i}\right) x_{d i s}\right] .
\end{gathered}
$$

A.4.2. Derivatives of $C_{d}(j, \boldsymbol{\theta})$ and $A_{d k}^{z}(j, \boldsymbol{\theta})$

Let us denote

$$
R_{d}\left(\boldsymbol{\theta}, j, v_{d}\right)=R_{d}(j)=\exp \left\{j \phi v_{d}-\sum_{i=1}^{n_{d}} \log \left[1+\exp \left\{\boldsymbol{x}_{d i} \boldsymbol{\beta}+\phi v_{d}\right\}\right]\right\} .
$$

Then $C_{d}$, defined in (3.1), can be written for $y_{d}=j$ in the form

$$
C_{d}(j, \boldsymbol{\theta})=C_{d}(j)=\int_{R} R_{d}\left(\boldsymbol{\theta}, j, v_{d}\right) f\left(v_{d}\right) d v_{d}
$$


and its first and second order partial derivatives are

$$
\begin{aligned}
\frac{\partial C_{d}(j)}{\partial \beta_{r}}= & -\int_{R} R_{d}(j)\left(\sum_{i=1}^{n_{d}} p_{d i} x_{d i r}\right) f\left(v_{d}\right) d v_{d}, \\
\frac{\partial^{2} C_{d}(j)}{\partial \beta_{s} \partial \beta_{r}}= & \int_{R} R_{d}(j)\left\{\left(\sum_{i=1}^{n_{d}} p_{d i} x_{d i r}\right)\left(\sum_{i=1}^{n_{d}} p_{d i} x_{d i s}\right)\right. \\
& \left.-\sum_{i=1}^{n_{d}} p_{d i}\left(1-p_{d i}\right) x_{d i r} x_{d i s}\right\} f\left(v_{d}\right) d v_{d} .
\end{aligned}
$$

The term $A_{d k}^{z}$, defined in (3.5), can be expressed for $y_{d .}=j$ and $q_{d k}$, given in (3.2), as

$$
A_{d k}^{z}(j, \boldsymbol{\theta})=A_{d k}^{z}(j)=\int_{R} q_{d k} R_{d}\left(\boldsymbol{\theta}, j, v_{d}\right) f\left(v_{d}\right) d v_{d}
$$

and its first and second order partial derivatives are

$$
\begin{aligned}
\frac{\partial A_{d k}^{z}(j)}{\partial \beta_{r}}= & \int_{R} q_{d k} R_{d}(j)\left\{\left(1-q_{d k}\right) z_{k r}-\sum_{i=1}^{n_{d}} p_{d i} x_{d i r}\right\} f\left(v_{d}\right) d v_{d} \\
\frac{\partial^{2} A_{d k}^{z}(j)}{\partial \beta_{s} \partial \beta_{r}}= & \int_{R} q_{d k}\left(1-q_{d k}\right) z_{k s} R_{d}(j)\left\{\left(1-q_{d k}\right) z_{k r}-\sum_{i=1}^{n_{d}} p_{d i} x_{d i r}\right\} f\left(v_{d}\right) d v_{d} \\
& -\int_{R} q_{d k} R_{d}(j)\left(\sum_{i=1}^{n_{d}} p_{d i} x_{d i s}\right)\left\{\left(1-q_{d k}\right) z_{k r}-\sum_{i=1}^{n_{d}} p_{d i} x_{d i r}\right\} f\left(v_{d}\right) d v_{d} \\
& +\int_{R} q_{d k} R_{d}(j)\left\{-q_{d k}\left(1-q_{d k}\right) z_{k r} z_{k s}-\sum_{i=1}^{n_{d}} p_{d i}\left(1-p_{d i}\right) x_{d i r} x_{d i s}\right\} f\left(v_{d}\right) d v_{d} .
\end{aligned}
$$

\section{A.4.3. Derivatives of $p_{d}(j, \boldsymbol{\theta})$}

From the definitions of $p_{d}(j, \boldsymbol{\theta})=p_{d}(j)$ in (4.1) and of $C_{d}(j, \boldsymbol{\theta})=C_{d}(j)$ in (A.3), it follows that

$$
p_{d}(j, \boldsymbol{\theta})=\sum_{\boldsymbol{y} \in S_{n_{d}, j}}\left[\exp \left\{\sum_{i=1}^{n_{d}} y_{i} \boldsymbol{x}_{d i} \boldsymbol{\beta}\right\} C_{d}(j, \boldsymbol{\theta})\right]
$$

So that the first- and second-order partial derivatives of $p_{d}(j)$ are

$$
\frac{\partial p_{d}(j)}{\partial \beta_{r}}=\sum_{\boldsymbol{y} \in S_{n_{d}, j}} \exp \left\{\sum_{i=1}^{n_{d}} y_{i} \boldsymbol{x}_{d i} \boldsymbol{\beta}\right\}\left[\left(\sum_{i=1}^{n_{d}} y_{i} x_{d i r}\right) C_{d}(j)+\frac{\partial C_{d}(j)}{\partial \beta_{r}}\right],
$$




$$
\begin{aligned}
\frac{\partial^{2} p_{d}(j)}{\partial \beta_{s} \partial \beta_{r}}= & \sum_{y \in S_{n_{d}, j}} \exp \left\{\sum_{i=1}^{n_{d}} y_{i} \boldsymbol{x}_{d i} \boldsymbol{\beta}\right\}\left[\left(\sum_{i=1}^{n_{d}} y_{i} x_{d i r}\right)\left(\sum_{i=1}^{n_{d}} y_{i} x_{d i s}\right) C_{d}(j)\right. \\
& \left.+\left(\sum_{i=1}^{n_{d}} y_{i} x_{d i r}\right) \frac{\partial C_{d}(j)}{\partial \beta_{s}}+\left(\sum_{i=1}^{n_{d}} y_{i} x_{d i s}\right) \frac{\partial C_{d}(j)}{\partial \beta_{r}}+\frac{\partial^{2} C_{d}(j)}{\partial \beta_{s} \partial \beta_{r}}\right]
\end{aligned}
$$

\section{A.4.4. Derivatives of $\psi_{d}\left(y_{d}, \boldsymbol{\theta}\right)$}

The first- and second-order partial derivatives of

$$
\psi_{d}(j, \boldsymbol{\theta})=\psi_{d}(j)=\sum_{k=1}^{K} N_{d k} \frac{A_{d k}^{z}(j)}{C_{d}(j)}
$$

defined in (3.4), are

$$
\begin{aligned}
\frac{\partial \psi_{d}(j, \boldsymbol{\theta})}{\partial \theta_{r}}=\sum_{k=1}^{K} N_{d k}\left\{\frac{\frac{\partial A_{d k}^{z}(j)}{\partial \theta_{r}}}{C_{d}(j)}-\frac{A_{d k}^{z}(j) \frac{\partial C_{d}(j)}{\partial \theta_{r}}}{C_{d}^{2}(j)}\right\}, \\
\frac{\partial^{2} \psi_{d}(j, \boldsymbol{\theta})}{\partial \theta_{s} \partial \theta_{r}}=\sum_{k=1}^{K} N_{d k}\left\{\frac{\frac{\partial^{2} A_{d k}^{z}(j)}{\partial \theta_{s} \partial \theta_{r}}}{C_{d}(j)}-\frac{\frac{\partial A_{d k}^{z}(j)}{\partial \theta_{r}} \frac{\partial C_{d}(j)}{\partial \theta_{s}}+\frac{\partial A_{d k}^{z}(j)}{\partial \theta_{s}} \frac{\partial C_{d}(j)}{\partial \theta_{r}}+A_{d k}^{z}(j) \frac{\partial^{2} C_{d}(j)}{\partial \theta_{s} \partial \theta_{r}}}{C_{d}^{2}(j)}\right. \\
\left.+\frac{2 A_{d k}^{z}(j) \frac{\partial C_{d}(j)}{\partial \theta_{s}} \frac{\partial C_{d}(j)}{\partial \theta_{r}}}{C_{d}^{3}(j)}\right\} .
\end{aligned}
$$

\section{A.4.5. Derivatives of $g_{d}(\boldsymbol{\theta})$}

We recall that

$$
g_{d}(\boldsymbol{\theta})=\int_{R}\left(\sum_{k=1}^{K} N_{d k} q_{d k}\left(\boldsymbol{\theta}, v_{d}\right)\right)^{2} f\left(v_{d}\right) d v_{d}-\sum_{j=0}^{n_{d}} \psi_{d}^{2}(j, \boldsymbol{\theta}) p_{d}(j, \boldsymbol{\theta}) .
$$

The first order partial derivatives of $g_{d}(\boldsymbol{\theta})$ are

$$
\begin{aligned}
\frac{\partial g_{d}(\boldsymbol{\theta})}{\partial \beta_{r}}= & 2 \int_{R}\left(\sum_{k=1}^{K} N_{d k} q_{d k}\right)\left(\sum_{k=1}^{K} N_{d k} q_{d k}\left(1-q_{d k}\right) z_{k r}\right) f\left(v_{d}\right) d v_{d} \\
& -2 \sum_{j=0}^{n_{d}} \psi_{d}(j) \frac{\partial \psi_{d}(j)}{\partial \beta_{r}} p_{d}(j)-\sum_{j=0}^{n_{d}} \psi_{d}^{2}(j) \frac{\partial p_{d}(j)}{\partial \beta_{r}} .
\end{aligned}
$$


The second order partial derivatives of $g_{d}(\boldsymbol{\theta})$ are

$$
\begin{aligned}
\frac{\partial^{2} g_{d}(\boldsymbol{\theta})}{\partial \beta_{s} \partial \beta_{r}} & =2 \int_{R}\left(\sum_{k=1}^{K} N_{d k} q_{d k}\left(1-q_{d k}\right) z_{k s}\right)\left(\sum_{k=1}^{K} N_{d k} q_{d k}\left(1-q_{d k}\right) z_{k r}\right) f\left(v_{d}\right) d v_{d} \\
& +2 \int_{R}\left(\sum_{k=1}^{K} N_{d k} q_{d k}\right)\left(\sum_{k=1}^{K} N_{d k} q_{d k}\left(1-q_{d k}\right)\left(1-2 q_{d k}\right) z_{k r} z_{k s}\right) f\left(v_{d}\right) d v_{d} \\
& -2 \sum_{j=0}^{n_{d}} \frac{\partial \psi_{d}(j)}{\partial \beta_{s}} \frac{\partial \psi_{d}(j)}{\partial \beta_{r}} p_{d}(j)-2 \sum_{j=0}^{n_{d}} \psi_{d}(j) \frac{\partial^{2} \psi_{d}(j)}{\partial \beta_{s} \partial \beta_{r}} p_{d}(j)-2 \sum_{j=0}^{n_{d}} \psi_{d}(j) \frac{\partial \psi_{d}(j)}{\partial \beta_{r}} \frac{\partial p_{d}(j)}{\partial \beta_{s}} \\
& -2 \sum_{j=0}^{n_{d}} \psi_{d}(j) \frac{\partial \psi_{d}(j)}{\partial \beta_{s}} \frac{\partial p_{d}(j)}{\partial \beta_{r}}-\sum_{j=0}^{n_{d}} \psi_{d}^{2}(j) \frac{\partial^{2} p_{d}(j)}{\partial \beta_{s} \partial \beta_{r}} .
\end{aligned}
$$

\section{A.4.6. Approximations of the Derivatives}

The integrals appearing in the described derivatives can be approximated by Monte Carlo simulation. To illustrate the procedure, we present the corresponding formulas used to approximate the derivatives of the term $C_{d}(j)$ derived in Section A.4.2. The approximations of the derivatives of the remaining terms can be done in a similar way.

For $s=1, \ldots, S$, let $v_{d}^{(s)}$ be i.i.d. $N(0,1)$ random variables and $v_{d}^{(S+s)}=-v_{d}^{(s)}$. The partial derivatives of $C_{d}(j)$ can be approximated as follows:

$$
\begin{aligned}
\frac{\partial \hat{C}_{d}(j)}{\partial \beta_{r}}= & -\frac{1}{2 S} \sum_{s=1}^{2 S} \hat{R}_{d}^{(s)}(j)\left(\sum_{i=1}^{n_{d}} \hat{p}_{d i}^{(s)} x_{d i r}\right), \\
\frac{\partial^{2} \hat{C}_{d}(j)}{\partial \beta_{s} \partial \beta_{r}}= & \frac{1}{2 S} \sum_{s=1}^{2 S} \hat{R}_{d}^{(s)}(j)\left\{\left(\sum_{i=1}^{n_{d}} \hat{p}_{d i}^{(s)} x_{d i r}\right)\left(\sum_{i=1}^{n_{d}} \hat{p}_{d i}^{(s)} x_{d i s}\right)\right. \\
& \left.-\sum_{i=1}^{n_{d}} \hat{p}_{d i}^{(s)}\left(1-\hat{p}_{d i}^{(s)}\right) x_{d i r} x_{d i s}\right\},
\end{aligned}
$$

where

$$
\hat{R}_{d}^{(s)}(j)=R\left(\hat{\boldsymbol{\theta}}, j, v_{d}^{(s)}\right) \quad \text { and } \quad \hat{p}_{d i}^{(s)}=\frac{\exp \left\{\boldsymbol{x}_{d i} \hat{\boldsymbol{\beta}}+\hat{\boldsymbol{\phi}} v_{d}^{(s)}\right\}}{1+\exp \left\{\boldsymbol{x}_{d i} \hat{\boldsymbol{\beta}}+\hat{\boldsymbol{\phi}} v_{d}^{(s)}\right\}}
$$

\section{References}

Chambers, R., N. Salvati, and N. Tzavidis. 2012. M-Quantile Regression for Binary Data with Application to Small Area Estimation. Working Paper 12-12, 2012, 24. Centre for Statistical and Survey Methodology, University of Wollongong. Available at: http://ro.uow.edu.au/cssmwp/101 (accessed January 2016). 
Elbers, C., J.O. Lanjouw, and P. Lanjouw. 2003. "Micro-level Estimation of Poverty and Inequality." Econometrica 71: 355-364. Doi: http://dx.doi.org/10.1111/14680262.00399.

Erciulescu, A.L. and W. Fuller. 2014. "Parametric Bootstrap Procedures for Small Area Prediction Variance.” In Proceedings of the Joint Statistical Meeting - Survey Research Methods Section, August 6, 2014, Boston. 3307-3318. Available at: https://www. amstat. org/sections/srms/proceedings/y2014/Files/31294890280.pdf (accessed January 2016).

Esteban, M.D., D. Morales, A. Pérez, and L. Santamaría. 2012a. "Two Area-Level Time Models for Estimating Small Area Poverty Indicators." Journal of the Indian Society of Agricultural Statistics 66: 75-89.

Esteban, M.D., D. Morales, A. Pérez, and L. Santamaría. 2012b. "Small Area Estimation of Poverty Proportions under Area-Level Time Models." Computational Statistics and Data Analysis 56: 2840-2855. Doi: http://dx.doi.org/10.1016/j.csda.2011.10.015.

Farrell, P., B. MacGibbon, and T. Tomberlin. 1997. "Bootstrap Adjustments for Empirical Bayes Interval Estimates of Small Area Proportions.” Canadian Journal of Statistics 25: 75-89. Doi: http://dx.doi.org/10.2307/3315358.

Ghosh, M. and J. Rao. 1994. “Small Area Estimation: An Appraisal.” Statistical Science 9: 55-93.

González-Manteiga, W., M.J. Lombardía, I. Molina, D. Morales, and L. Santamaría. 2007. "Estimation of the Mean Squared Error of Predictors of Small Area Linear Parameters under a Logistic Mixed Model." Computational Statistics and Data Analysis 51: 2720-2733. Doi: http://dx.doi.org/10.1016/j.csda.2006.01.012.

González-Manteiga, W., M.J. Lombardía, I. Molina, D. Morales, and L. Santamaría. 2008a. "Bootstrap Mean Squared Error of Small-Area EBLUP." Journal of Statistical Computation and Simulation 78: 443-462. Doi: http://dx.doi.org/10.1080/0094 9650601141811.

González-Manteiga, W., M.J. Lombardía, I. Molina, D. Morales, and L. Santamaría. 2008b. "Analytic and Bootstrap Approximations of Prediction Errors under a Multivariate Fay-Herriot Model." Computational Statistics and Data Analysis 52: 5242-5252. Doi: http://dx.doi.org/10.1016/j.csda.2008.04.031.

Hall, P. and T. Maiti. 2006a. "Nonparametric Estimation of Mean-Squared Prediction Error in Nested-Error Regression Models.” The Annals of Statistics 34: 1733-1750. Doi: http://dx.doi.org/10.1214/009053606000000579.

Hall, P. and T. Maiti. 2006b. "On Parametric Bootstrap Methods for Small Area Prediction." Journal of the Royal Statistical Society, Series B 68: 221-238. Doi: http://dx.doi.org/10.1111/j.1467-9868.2006.00541.x.

Jiang, J. 1998. “Consistent Estimators in Generalized Linear Models.” Journal of the American Statistical Association 93: 720-729. Doi: http://dx.doi.org/10.2307/2670122. Jiang, J. 2003. "Empirical Best Prediction for Small-Area Inference Based on Generalized Linear Mixed Models.” Journal of Statistical Planning and Inference 111: 117-127. Doi: http://dx.doi.org/10.1016/S0378-3758(02)00293-8.

Jiang, J. and P. Lahiri. 2001. "Empirical Best Prediction for Small Area Inference with Binary Data." Annals of the Institute of Statistical Mathematics 53: 217-243. Doi: http://dx.doi.org/10.1023/A:1012410420337. 
Jiang, J. and P. Lahiri. 2006. "Mixed Model Prediction and Small Area Estimation." Test 15: 1-96. Doi: http://dx.doi.org/10.1007/BF02595419.

Malec, D., J. Sedransk, C. Moriarity, and F. LeClere. 1997. "Small Area Inference for Binary Variables in the National Health Interview Survey." Journal of the American Statistical Association 92: 815-826. Doi: http://dx.doi.org/10.1080/01621459. 1997.10474037.

Marhuenda, Y., I. Molina, and D. Morales. 2013. "Small Area Estimation with SpatioTemporal Fay-Herriot Models." Computational Statistics and Data Analysis 58: 308-325. Doi: http://dx.doi.org/10.1016/j.csda.2012.09.002.

Molina, I., B. Nandram, and J.N.K. Rao. 2014. "Small Area Estimation of General Parameters with Application to Poverty Indicators: A Hierarchical Bayes Approach." The Annals of Applied Statistics 8: 852-885. Doi: http://dx.doi.org/10.1214/13-AOAS702. Molina, I. and J.N.K. Rao. 2010. "Small Area Estimation of Poverty Indicators." The Canadian Journal of Statistics 38: 369-385. Doi: http://dx.doi.org/10.1002/cjs.10051. Morales, D., M.C. Pagliarella, and R. Salvatore. 2015. "Small Area Estimation of Poverty Indicators under Partitioned Area-Level Time Models." SORT - Statistics and Operations Research Transactions 39: 19-34.

Pfeffermann, D. 2002. "Small Area Estimation - New Developments and Directions." International Statistical Review 70: 125-143. Doi: http://dx.doi.org/10.2307/1403729. Pfeffermann, D. 2013. "New Important Developments in Small Area Estimation." Statistical Science 28: 1-134. Doi: http://dx.doi.org/10.1214/12-STS395.

Rao, J.N.K. 1999. "Some Recent Advances in Model-Based Small Area Estimation." Survey Methodology 25: 175-186.

Rao, J.N.K. 2003. Small Area Estimation. New York: Wiley.

Rao, J.N.K. and I. Molina. 2015. Small Area Estimation, 2nd ed. New York: Wiley.

Received February 2015

Revised January 2016

Accepted May 2016 